\title{
POSITIVE SOLUTIONS FOR ASYMPTOTICALLY LINEAR PROBLEMS IN EXTERIOR DOMAINS
}

\author{
LILIANE A. MAIA AND BENEDETTA PELLACCI
}

\begin{abstract}
The existence of a positive solution for a class of asymptotically linear problems in exterior domains is established via a linking argument on the Nehari manifold and by means of a barycenter function.
\end{abstract}

\section{INTRODUCTION}

In this paper we look for a positive solution for the following class of elliptic problems

$$
\left\{\begin{array}{l}
-\Delta u+\lambda u=f(u), \quad \text { in } \Omega, \\
u \in H_{0}^{1}(\Omega)
\end{array}\right.
$$

where $\Omega$ is an exterior domain, i.e. a unbounded smooth domain in $\mathbb{R}^{N}, N \geq 2$, whose complement $\mathbb{R}^{N} \backslash \Omega$ is bounded and not empty. The non-linearity $f$ is superlinear at zero and asymptotically linear at infinity, and the model example we have in mind is

$$
f(t)=\frac{t^{3}}{1+s t^{2}}, \quad 0<s<\frac{1}{\lambda} .
$$

This kind of equations arises in the study of the propagation of a light beam in saturable media, as photorefractive crystals, which are largely exploited in experimental observations because they require low optical power to exhibit very strong non-linear effects $([3,26,32,35])$. When the medium has some additional properties ([2]), the non-linearity given in (1.2) is approximated by putting $s=0$ and obtaining $f(t)=t^{3}$, that is the more treated non-linearity, also associated to the study of the propagation of a light beam in the so-called Kerr media ([3]). From a mathematical point of view, this kind of problems has been extensively studied in

1991 Mathematics Subject Classification. 35J20, 35J25, 35J61, 35Q55.

Key words and phrases. Asymptotically linear problems, exterior domains, Schrödinger equation.

Research of the first author is partially supported by CNPq/Brazil, PROEX/CAPES and FAPDF193.000.939/2015.

Research of the second author is partially supported by MIUR-PRIN-2012-74FYK7 Grant: "Variational and perturbative aspects of nonlinear differential problems", by the Italian Project FIRB 2012: "Dispersive dynamics: Fourier Analysis and Variational Methods", "Gruppo Nazionale per l'Analisi Matematica, la Probabilità e le loro Applicazioni" (GNAMPA) of the Istituto Nazionale di Alta Matematica (INdAM); University Project "Sostegno alla ricerca individuale per il trienno 2015-2017”. 
the last thirty years, especially when $f(t)=|t|^{p-2} t$, and $p>2$ is subcritical. The main difficulty relies in the lack of compactness that can be overcome if $\Omega$ is radially symmetric and one looks for radially symmetric solutions ([10, 22]). While, if $\Omega$ has no symmetry properties, the lack of compactness becomes prevalent, moreover, it has been observed in [9] that there are no least action solutions, so that a higher action level solution has to be searched. As a consequence, a careful analysis of a general Palais-Smale sequence, even not minimising, is needed and this has been done in [9] introducing the nowadays so-called "splitting lemma". This analysis permits to locate the action levels where the Palais-Smale condition holds, so that, the difficulty becomes to construct a minimax level where this compactness property is satisfied. In [9] suitable subspaces of the $L^{p}(\Omega)$ sphere have been considered, with additional prescriptions, in order to increase the minimax level. This approach led to the existence of a constrained minimisation point, which, thanks to the homogeneity property of $f(t)=|t|^{p-2} t$ gives a positive solution when $\mathbb{R}^{N} \backslash \Omega$ has small diameter. This kind of argument has been developed in several directions: in [7] the assumption on the size of $\mathbb{R}^{N} \backslash \Omega$ has been removed, in [17, 18] multiplicity results have been obtained, in dependence on $\lambda$ and on the topology of $\Omega$ (see also the interesting survey [14]); in [15, 20] sign-changing solutions have been found. When $f$ has not a polynomial dependence with respect to $t$, there are only a few contributions in the literature. In particular, in the case in which $f$ is asymptotically linear, in [19] the existence of a solution is proved for $f$ depending on the spatial variable and satisfying some assumptions corresponding, in the autonomous case, to a convexity property. More recently, Problem (1.1) has been studied in [24] where it is found a positive solution under some suitable hypotheses on $f$, among which, again the convexity for $t \in(0,+\infty)$ and in [27], where it is proved the existence of a radial positive solution in the exterior of a ball.

Notice that, $f$ given in (1.2) is not convex so that the result in [19,24] do not cover the physical meaningful case. Moreover, we do not suppose any symmetry property on the domain, so that there is no reason to expect a radially symmetric solution. With this respect, our result in the model case (1.2) is related to the interest in the physical context of asymmetric solutions that are observed in the interaction between the crystal and the spatial solitons or in the collision of symmetric solitons $([3,26])$.

Our existence result in the model case can be stated as follows.

Theorem 1.1. Assume $N \geq 2$, and that $f$ is given in (1.2). Then, Problem (1.1) has at least a positive solution.

This theorem will follow as a consequence of a more general Theorem 2.2, proved for a general non-linearity $f(t)$, (see Section 2).

As in the super-linear case, when $f(t)$ is given in (1.2), one expects that the least action level of the associated functional $I_{\Omega}: H_{0}^{1}(\Omega) \mapsto \mathbb{R}$

$$
I_{\Omega}(u):=\int_{\Omega} \frac{1}{2}\left[|\nabla u|^{2}+\lambda u^{2}\right]-F(u), \quad \text { with } F(u)=\int_{0}^{u} f(t) d t
$$


is equal to the least action level of the functional associated to the problem in the whole $\mathbb{R}^{N}$

$$
I(u):=\int_{\mathbb{R}^{N}} \frac{1}{2}\left[|\nabla u|^{2}+\lambda u^{2}\right]-F(u),
$$

which is showed to be attained by a unique, positive, radially symmetric solution $([10,11,34])$. But, when dealing with asymptotically linear $f(t)$, even in the model case (1.2), one loses the precious homogeneity property, crucial in order to reabsorb the Lagrange multiplier when finding the least action solution as a constrained minimum point on a $L^{p}(\Omega)$ sphere. So that, working on $L^{p}(\Omega)$ spheres is not suitable in this context. In the light of these considerations, we will work on the well-known Nehari manifolds associated to the functionals $I_{\Omega}$ and $I([29,30])$

$$
\begin{aligned}
\mathcal{N}_{\Omega} & =\left\{u \in H_{0}^{1}(\Omega) \backslash\{0\}:\left\langle I_{\Omega}^{\prime}(u), u\right\rangle=0\right\}, \\
\mathcal{N} & =\left\{u \in H^{1}\left(\mathbb{R}^{N}\right) \backslash\{0\}:\left\langle I^{\prime}(u), u\right\rangle=0\right\},
\end{aligned}
$$

realizing the least action levels as minimum values of $I_{\Omega}$ and $I$ on these manifolds

$$
m_{\Omega}:=\inf _{u \in \mathcal{N}_{\Omega}} I_{\Omega}(u), \quad m:=\inf _{u \in \mathcal{N}} I(u) .
$$

This approach yields some new difficulties and some new advantages; concerning the formers, notice that not every function in $H_{0}^{1}(\Omega)$ can be projected on $\mathcal{N}_{\Omega}$, so that, one has to be careful when defining the projection of a function on $\mathcal{N}_{\Omega}$; But, thanks to a monotonicity condition of $f((2.3))$ the projection turns out to be unique when it exists. With this approach we will show that $m_{\Omega}=m$ so that a higher energy critical level has to be searched also in this case. On the other hand, we have some benefits when looking for a compactness property; indeed, recall that, usually when dealing with asymptotically linear non-linearity the suitable notion of compactness property is the Cerami condition introduced in [13]; here, strongly exploiting the features of $\mathcal{N}_{\Omega}$, we will be able to show that the usual Palais-Smale condition on $\mathcal{N}_{\Omega}$ holds. However, in doing this, we will again have to handle the lack of homogeneity of $f$.

Finally, we will be able to choose linking sets as proper subsets of $\mathcal{N}_{\Omega}$. At this stage, it will be important to show proper estimates of $I_{\Omega}$ on these subsets. This will be done by introducing a suitable abstract asymptotic threshold (in Lemma 3.2 ), that will be crucial in order to compare all the terms in the functional $I_{\Omega}$ (defined in (1.3)). This threshold generalises the commonly used one in the context of polynomial non-linearity. Concluding, let us observe that, in order to highlight the main novelties and difficulties one encounters when facing this kind of problems, we have preferred to study the existence of a solution, but we believe that with the same tools introduced here it could be possible to face the question of multiplicity of solutions as done in [16, 18] for the polynomial case. Moreover, our argument can be used to prove an analogous result for a general, not necessarily homogeneous, super-linear $f$ (see for more details Remark 2.4).

Concluding this introduction, let us point out that the use of the constraint of $\mathrm{Ne}$ hari manifold has extensively been used in the related context of the problem in the whole space with the presence of a potential (see for instance $[5,39,38]$ and 
the references therein). In particular, in $[38,23]$ the properties of a generalized $\mathrm{Ne}$ hari manifold are deeply exploited in order to study indefinite problems; here we choose to assume all the regularity conditions needed to use the classical Nehari manifold, but it would be interesting to use the analysis of $[38,23]$ to weaken our regularity assumptions on the non-linearity.

The paper is organized as follows: in Section 2 we settle our general context and state our main existence result (Theorem 2.2); in Section 3 we prove some useful technical propositions, while in Section 4 we deal with the compactness property. Finally, in Section 5 we conclude the proofs of Theorem 2.2 and 1.1.

\section{Setting of THE PROBLEM AND MAIN RESUltS}

We will work in $H_{0}^{1}(\Omega)$ and in $H^{1}\left(\mathbb{R}^{N}\right)$. The norms on these spaces will be denoted respectively by

$$
\|u\|_{\Omega}^{2}=(u, u)_{\Omega}=\|\nabla u\|_{2, \Omega}^{2}+\lambda\|u\|_{2, \Omega}^{2}, \quad\|u\|^{2}=(u, u)=\|\nabla u\|_{2}^{2}+\lambda\|u\|_{2}^{2},
$$

where $\|\cdot\|_{p . \Omega}\left(\|\cdot\|_{p}\right)$ is the usual norm in $L^{p}(\Omega)\left(\right.$ in $\left.L^{p}\left(\mathbb{R}^{N}\right)\right)$.

Every solution of (1.1), is a critical point of the $C^{1}$ functional $I_{\Omega}$, defined in (1.3). We have introduced the model example in (1.2), however, in general, we will assume that $f$ has the following properties

$$
f \in C^{1}[0,+\infty) \cap C^{3}(0,+\infty), f(t) \equiv 0, \forall t \in(-\infty, 0) ;
$$

there exists a positive constant $D$ and $p_{1} \leq p_{2} \in(1, \bar{p})$ where $\bar{p}=+\infty$ if $N=2$ and $\bar{p}=(N+2) / N-2$ if $N \geq 3$, such that

$$
\left|f^{(k)}(t)\right| \leq D\left[|t|^{p_{1}-k}+|t|^{p_{2}-k}\right], k=0,1,2,3 .
$$

The following assumption will be crucial in all our arguments:

$$
\frac{f(t)}{t} \text { is an increasing function } \forall t>0 \text {. }
$$

Moreover, we suppose that $f$ is asymptotically linear at infinity, namely, we assume that there exists a positive number $l_{\infty}$ such that

$$
\lim _{t \rightarrow+\infty} \frac{f(t)}{t}=l_{\infty}, \quad \lambda-l_{\infty}<0,
$$

where the second inequality is assumed in order to have a solution of the problem in the whole $\mathbb{R}^{N}$ (see $[10,11,36]$ ). When dealing with this kind of non-linearities it is often assumed a non-quadraticity type condition (introduced in [21])

$$
f(t) t-2 F(t) \geq 0, \text { for all } t \in \mathbb{R}^{+} \text {and } \lim _{t \rightarrow+\infty}[f(t) t-2 F(t)]=+\infty .
$$

Finally, in order to let the Nehari manifold be a natural constraint, we will assume that

$$
f^{\prime}(t)-\frac{f(t)}{t}>0 \text { for every } t>0 .
$$

Remark 2.1. Notice that all the previous hypotheses are satisfied in the model case (1.2). Moreover note that, as a consequence of (2.3) and (2.4), the general non-linearity $f(t)$ is always below the line $l_{\infty} t$, for every $t>0$. 
In order to prove our existence result we will make a comparison with the following problem in the whole $\mathbb{R}^{N}$ which will be called problem at infinity

$$
\left\{\begin{array}{l}
-\Delta u+\lambda u=f(u), \quad \text { in } \quad \mathbb{R}^{N}, \\
\lim _{|x| \rightarrow \infty} u(x)=0 .
\end{array}\right.
$$

The problem (2.7) has a positive, radially symmetric, least action solution (whose existence is proved in [10,36] for $N \geq 3$ and in [11] for $N=2$.), which we denote with $w$. In [34] it is shown the uniqueness of $w$ when $f$ satisfies the additional hypothesis

$$
g(t):=\frac{-\lambda t+t f^{\prime}(t)}{-\lambda t+f(t)} \text { is decreasing in }[b,+\infty)
$$

where $b$ is the (unique thanks to (2.3)) positive number given by $f(b)=\lambda b$. Notice that this condition is satisfied in the case of (1.2). Since we will also deal with a more general non-linearity and will not assume this hypothesis we will suppose that

$(U) \quad$ The positive radially symmetric solution of problem (2.7) is unique.

Our main result is the following.

Theorem 2.2. Assume $N \geq 2$ and hypotheses (2.1), (2.2), (2.3), (2.4), (2.5), (2.6) and $(U)$. Then Problem (1.1) has at least a positive solution.

Remark 2.3. In [19] Problem (1.1) is studied for a non-autonomous $f$ satisfying some suitable hypotheses including one, that becomes, in the autonomous case, a convexity condition. In [24] Problem (1.1) is studied assuming (2.1), (2.3), (2.4), (2.5), (2.6), $(U)$ plus other conditions among which it is assumed that $\mathrm{f}$ is a convex function. Note that, $f$ given in (1.2) is not convex, so that the existence of a positive solution in exterior domains for this kind of non-linearity cannot be deduced from the result of $[19,24]$. Obviously, our result applies also to non-linearities that have infinitely many flex points.

Remark 2.4. Our argument can be exploited to deal with super-linear, not necessarily homogeneous, non-linearities assuming conditions (2.1), (2.2), (2.3), (2.6) and, in place of (2.4) and (2.5), supposing the classical Ambrosetti-Rabinowitz condition

$$
\exists \mu>2,: 0<\mu F(t) \leq t f(t), \quad \forall t \in \mathbb{R}^{+} \backslash\{0\} .
$$

Indeed, also under these hypotheses, it is possible to exploit the properties of the Nehari manifold $\mathcal{N}_{\Omega}$ as explained in [33].

As already said in the introduction, we will work on the Nehari manifold introduced in (1.4). Nowadays, this has become a classical tool in variational methods thanks of its useful features as it has been also highlighted in the recent contribution [31]. The following remark clarifies the role of (2.6) in order to use $\mathcal{N}_{\Omega}$. 
Remark 2.5. Let us first observe that $\mathcal{N}_{\Omega}$ is the set of non-trivial zeroes of the function $N_{\Omega}: H_{0}^{1}(\Omega) \backslash\{0\} \mapsto \mathbb{R}$ given by

$$
N_{\Omega}(u)=\left\langle I_{\Omega}^{\prime}(u), u\right\rangle=\|u\|_{\Omega}^{2}-\int_{\Omega} f(u) u .
$$

Notice that $\mathcal{N}_{\Omega}$ is actually a manifold and it is a natural constraint. Namely, for every $u \in \mathcal{N}_{\Omega}$ it results $\left\langle N_{\Omega}^{\prime}(u), u\right\rangle<0$. Indeed, consider $u \in \mathcal{N}_{\Omega}$ and use (2.2) and (2.6) to obtain

$$
\left\langle N_{\Omega}^{\prime}(u), u\right\rangle=2\|u\|^{2}-\int_{\Omega}\left[f^{\prime}(u) u^{2}+f(u) u\right]=\int_{\Omega} u^{2}\left[\frac{f(u)}{u}-f^{\prime}(u)\right]<0 .
$$

Now, suppose that $u \in \mathcal{N}_{\Omega}$ is a constrained critical point of $I_{\Omega}$, then there exists a real number $\mu$ such that $I_{\Omega}^{\prime}(u)-\mu N_{\Omega}^{\prime}(u)=0$; taking $u$ as test function one gets $\mu\left\langle N_{\Omega}^{\prime}(u), u\right\rangle=0$ then yields $\mu=0$, i.e. $u$ is a free critical point.

\section{Asymptotic Estimates}

In the sequel, when we compute the $H^{1}\left(\mathbb{R}^{N}\right)$ norm of a function $u \in H_{0}^{1}(\Omega)$, it is implicitly assumed that $u$ is extended as zero outside $\Omega$ so that $u \in H^{1}\left(\mathbb{R}^{N}\right)$. Let us introduce a cut-off $C^{\infty}$ function $\xi: \mathbb{R}^{N} \rightarrow[0,1]$ defined by

$$
\xi(x):=\tilde{\xi}\left(\frac{|x|}{\rho}\right),
$$

with $\rho$ being the smallest positive number such that $\mathbb{R}^{N} \backslash \Omega \subset B_{\rho}(0)$ and $\tilde{\xi}: \mathbb{R}^{+} \cup$ $\{0\} \rightarrow[0,1]$ being a non decreasing function such that

$$
\tilde{\xi}(t)=0 \quad \text { if } \quad t \leq 1 \quad \text { and } \quad \tilde{\xi}(t)=1 \quad \text { if } \quad t \geq 2, \quad\left|\tilde{\xi}^{\prime}(t)\right| \leq 2 .
$$

Recall that $w$, the least action positive solution of Problem (2.7) enjoys the following asymptotic behavior (see [10])

$$
\lim _{R \rightarrow+\infty} w(R) R^{(N-1) / 2} e^{\sqrt{\lambda} R}=\sigma, \quad \lim _{R \rightarrow+\infty} w^{\prime}(R) R^{(N-1) / 2} e^{\sqrt{\lambda} R}=-\sigma \sqrt{\lambda},
$$

we will often use the following Lemma proved in Lemma II.2 in [7], (see also Proposition 1.2 in [6]).

Lemma 3.1. Let $\varphi_{1} \in C\left(\mathbb{R}^{N}\right) \cap L^{\infty}\left(\mathbb{R}^{N}\right), \varphi_{2} \in C\left(\mathbb{R}^{N}\right)$ satisfy for some $\alpha, \beta \geq 0$, $c \in \mathbb{R}$

$$
\lim _{|x| \rightarrow+\infty} \varphi_{1}(x) e^{\alpha|x|}|x|^{\beta}=c, \quad \int_{\mathbb{R}^{N}}\left|\varphi_{2}(x)\right| e^{\alpha|x|}\left(1+|x|^{\beta}\right) d x<+\infty,
$$

then

$$
\lim _{|\zeta| \rightarrow+\infty} e^{\alpha|\zeta|}|\zeta|^{\beta} \int_{\mathbb{R}^{N}} \varphi_{1}(x+\zeta) \varphi_{2}(x) d x=c \int_{\mathbb{R}^{N}} \varphi_{2}(x) d x .
$$

In what follows we will use the notation

$$
w^{\theta}(\cdot):=w(\cdot-\theta), \quad \forall \theta \in \mathbb{R}^{N} \backslash\{0\} .
$$

As a consequence of the previous lemma, it is easy to prove the following result. 
Lemma 3.2. Assume (2.2) and let $x_{0} \in \mathbb{R}^{N}$ with $\left|x_{0}\right|=1$ and $y \in \partial B_{2}\left(x_{0}\right)$. Let us define the quantity

$$
\varepsilon_{R}:=\int_{\mathbb{R}^{N}} f\left(w^{R x_{0}}\right) w^{R y}=\int_{\mathbb{R}^{N}} f\left(w^{R y}\right) w^{R x_{0}} .
$$

Then, $\varepsilon_{R}$ satisfies

$$
\lim _{R \rightarrow+\infty} \varepsilon_{R}\left[(2 R)^{(N-1) / 2} e^{2 R \sqrt{\lambda}}\right]=c_{0}>0 .
$$

Remark 3.3. Let us observe that, in [23] an analogous asymptotic threshold has been introduced in order to deduce precise energy estimates, for an indefinite problem in the whole space with super-linear, but not necessarily homogeneous, nonlinearities.

Proof. The proof can be done following [6] or [7]; indeed by a change of variable, it results

$$
\varepsilon_{R}=\int_{\mathbb{R}^{N}} f(w) w^{R\left(x_{0}-y\right)} .
$$

In order to obtain the conclusion it is sufficient to apply Lemma 3.1 with $\varphi_{1}=$ $w, \varphi_{2}=f(w)$ and $\zeta=-R\left(x_{0}-y\right)$. Let us check that all the hypotheses are satisfied. Using (3.3), we obtain that $\varphi_{1}$ satisfies the hypothesis of Lemma 3.1 with $\alpha=\sqrt{\lambda}$ and $\beta=(N-1) / 2$. Let us see what happens with $\varphi_{2}$. The first limit in (3.3) and condition (2.2) imply that there exists $R_{1}$ such that

$$
\varphi_{2}(x)=f(w) \leq 2 D \sigma\left[e^{-\alpha p_{1}|x|}|x|^{-\beta p_{1}}+e^{-\alpha p_{2}|x|}|x|^{-\beta p_{2}}\right] \quad \forall|x|>R_{1} .
$$

Then it holds, with $C$ a positive constant

$$
\begin{aligned}
\int_{\mathbb{R}^{N}} f(w(x)) e^{\alpha|x|}(1+|x|)^{\beta} & \leq C \operatorname{meas} B_{R_{1}}(0)+D C \int_{\left\{|x|>R_{1}\right\}} e^{\left(1-p_{1}\right) \alpha|x|}|x|^{-\beta p_{1}}\left(1+|x|^{\beta}\right) \\
& +D C \int_{\left\{|x|>R_{1}\right\}} e^{\left(1-p_{2}\right) \alpha|x|}|x|^{-\beta p_{2}}\left(1+|x|^{\beta}\right)
\end{aligned}
$$

so that the hypotheses of Lemma 3.1 are satisfied since $p_{i}>1$.

Moreover, for every $q>1$, for every $\theta \in \mathbb{R}^{N} \backslash\{0\}$ and for every compact set $K$ Lemma 3.1 implies

$$
\int_{K}\left[w^{R \theta}\right]^{q} \leq O\left(\frac{1}{R^{(N-1) q / 2} e^{R q \sqrt{\lambda}}}\right) .
$$

Indeed, denote with $a$ the modulus of $\theta$ and observe that (3.3) implies that there exists $\rho_{0}$ such that it results

$$
w(z)(|z|)^{(N-1) / 2} e^{\sqrt{\lambda}|z|} \leq 2 \sigma, \quad \text { for every }|z| \geq \rho_{0} .
$$

Let us fix $r_{0}$ such that $K \subseteq B_{r_{0}}(0)$ and put $z=x-R \theta$. We have

$$
R^{(N-1) q / 2} e^{R q \sqrt{\lambda}} \int_{K}\left[w^{R \theta}\right]^{q} \leq R^{(N-1) q / 2} e^{R q \sqrt{\lambda}} \int_{B_{r_{0}}(R \theta)}[w(z)]^{q} d z
$$


Moreover, $|z| \geq R a-r_{0}$, so that, for every $R$ such that $R a-r_{0}>\rho_{0}$, inequality (3.6) implies that there exists a positive constant $C$ such that

$$
R^{(N-1) q / 2} e^{R q \sqrt{\lambda}} \int_{K}\left[w^{R \theta}\right]^{q} \leq(2 \sigma)^{q} C .
$$

Therefore, for every $q \geq 2$, for every $\theta \in \mathbb{R}^{N} \backslash\{0\}$ and for every compact set $K$, it results

$$
\int_{K}\left[w^{R \theta}\right]^{q} \leq o\left(\varepsilon_{R}\right), \quad \int_{K}\left[\left|\nabla w^{R \theta}\right|\right]^{q} \leq o\left(\varepsilon_{R}\right),
$$

where the second inequality follows by an analogous argument for $w^{\prime}$. For every $\theta \in \mathbb{R}^{N} \backslash\{0\}$ let us define the map $\Phi^{\theta}: \mathbb{R}^{+} \rightarrow H_{0}^{1}(\Omega)$ by

$$
R \mapsto \Phi^{\theta}(R)(\cdot):=\xi(\cdot) w(\cdot-R \theta) .
$$

In the sequel, for simplicity, we will use the notation

$$
\Phi^{R \theta}:=\Phi^{\theta}(R)
$$

Lemma 3.4. Let us assume (2.1) and (2.2). Let $\theta \in \mathbb{R}^{N} \backslash\{0\}$. Then, the function $\Phi^{R \theta}$ is continuous in $R$ and

$$
\begin{gathered}
\left\|\Phi^{R \theta}-w^{R \theta}\right\|^{2} \leq o\left(\varepsilon_{R}\right), \quad\left|\left\|\Phi^{R \theta}\right\|^{2}-\left\|w^{R \theta}\right\|^{2}\right| \leq o\left(\varepsilon_{R}\right), \\
\left|I_{\Omega}\left(\Phi^{R \theta}\right)-I(w)\right| \leq o\left(\varepsilon_{R}\right) .
\end{gathered}
$$

Moreover, for every $\tau \geq 0$ it results

$$
\left|\int_{\mathbb{R}^{N}} F\left(\tau \Phi^{R \theta}\right)-F\left(\tau w^{R \theta}\right)\right| \leq\left[\tau^{p_{1}+1}+\tau^{p_{2}+1}\right] o\left(\varepsilon_{R}\right) .
$$

Proof. The continuity with respect to $R$ of $\Phi^{R \theta}$ follows from the regularity properties of $w$. Moreover, taking into account (3.2), (3.4) and using Young inequality, it follows

$$
\begin{aligned}
\left\|\Phi^{R \theta}-w^{R \theta}\right\|^{2}= & \int_{\mathbb{R}^{N}}\left|\nabla \xi w^{R \theta}+(\xi-1) \nabla w^{R \theta}\right|^{2}+\lambda \int_{\mathbb{R}^{N}}|\xi-1|^{2}\left(w^{R \theta}\right)^{2} \\
\leq & \int_{B_{2 \rho}(0)}\left(w^{R \theta}\right)^{2}\left[|\nabla \xi|^{2}|+\lambda| \xi-\left.1\right|^{2}\right]+\int_{B_{2 \rho}(0)}|\xi-1|^{2}\left|\nabla w^{R \theta}\right|^{2} \\
& +2 \int_{B_{2 \rho}(0)} w^{R \theta}|\xi-1||\nabla \xi|\left|\nabla w^{R \theta}\right| \\
\leq & \int_{B_{2 \rho}(0)}\left(w^{R \theta}\right)^{2}\left[2|\nabla \xi|^{2}|+\lambda| \xi-\left.1\right|^{2}\right]+2 \int_{B_{2 \rho}(0)}|\xi-1|^{2}\left|\nabla w^{R \theta}\right|^{2} \\
\leq & 2 \int_{B_{2 \rho}(0)}|\nabla w(x-R \theta)|^{2} d x+C \int_{B_{2 \rho}(0)}|w(x-R \theta)|^{2} d x,
\end{aligned}
$$

with $C$ a positive constant. Then, (3.9) follows from (3.7).

In order to show the second conclusion in (3.9), note that (3.2) and (3.7) imply

$$
\left|\left(w^{R \theta}, \Phi^{R \theta}-w^{R \theta}\right)\right| \leq \int_{B_{2 \rho}(0)} 3\left|\nabla w^{R \theta}\right|^{2}+2\left(w^{R \theta}\right)^{2} \leq o\left(\varepsilon_{R}\right)
$$


this and the first conclusion in (3.9) yield the second one.

To prove (3.10), let us observe that $I_{\Omega}\left(\Phi^{y}\right)=I\left(\Phi^{y}\right)$. Therefore, from (3.2), (2.2), and using Lagrange mean value Theorem, we get that there exists a positive constant $C_{0}$ such that

$$
\begin{aligned}
\left|I_{\Omega}\left(\Phi^{R \theta}\right)-I(w)\right| & \leq||\left|\Phi^{R \theta}\left\|^{2}-\right\| w^{R \theta} \|^{2}\right|+\left|\int_{\mathbb{R}^{N}} F\left(\xi w^{R \theta}\right)-F\left(w^{R \theta}\right)\right| \\
& \leq||\left|\Phi^{R \theta}\left\|^{2}-\right\| w^{R \theta} \|^{2}\right|+C_{0} D \int_{B_{2 \rho}(0)}\left(w^{R \theta}\right)^{p_{1}+1}+\left(w^{R \theta}\right)^{p_{2}+1} .
\end{aligned}
$$

Then, (3.10) follows from (3.7) and (3.9). Moreover, taking into account (3.2) and (3.4), and using condition (2.2) we get that there exists a positive constant $C_{1}$ such that

$$
\left|\int_{\mathbb{R}^{N}} F\left(\tau w^{R \theta}\right)-F\left(\tau \Phi^{R \theta}\right)\right| \leq C_{1} D \int_{B_{2 \rho}(0)} \tau^{p_{1}+1}\left(w^{R \theta}\right)^{p_{1}+1}+\tau^{p_{2}+1}\left(w^{R \theta}\right)^{p_{2}+1} .
$$

Then the conclusion follows from (3.7).

As already said, the linking sets will be subsets of $\mathcal{N}_{\Omega}$, but, as $f$ is asymptotically linear, not every function of $H_{0}^{1}(\Omega)$ can be projected on $\mathcal{N}_{\Omega}$. The following lemma tells us when this projection can be performed.

Lemma 3.5. Let us assume (2.1), (2.3), (2.4), and define the maps

$$
\mathcal{G}_{\Omega}(u)=\|\nabla u\|_{2, \Omega}^{2}+\lambda\|u\|_{2, \Omega}^{2}-l_{\infty}\left\|u^{+}\right\|_{2, \Omega}^{2}, \quad \mathcal{G}(u)=\|\nabla u\|_{2}^{2}+\lambda\|u\|_{2}^{2}-l_{\infty}\left\|u^{+}\right\|_{2}^{2} .
$$

Then, the maps

$$
\begin{aligned}
T_{\Omega}: A_{\Omega}:=\left\{u \in H_{0}^{1}(\Omega): \mathcal{G}_{\Omega}(u)<0\right\} & \mapsto \mathbb{R}^{+}, \quad u \mapsto T_{\Omega}(u): T_{\Omega}(u) u \in \mathcal{N}_{\Omega}, \\
T: A:=\left\{u \in H^{1}\left(\mathbb{R}^{N}\right): \mathcal{G}(u)<0\right\} & \mapsto \mathbb{R}^{+}, \quad u \mapsto T(u): T(u) u \in \mathcal{N}
\end{aligned}
$$

are well defined and continuous. In addition, for every $\theta \in \mathbb{R}^{N} \backslash\{0\}$, there exists $R_{0}=R_{0}(|\theta|)$ such that $\Phi^{R \theta} \in A_{\Omega} \cap A$ for every $R \geq R_{0}$ and

$$
\lim _{R \rightarrow+\infty} T_{\Omega}\left(\Phi^{R \theta}\right)=1 \text {. }
$$

Proof. Let us define $g_{\Omega}:[0,+\infty) \times A_{\Omega} \mapsto \mathbb{R}$ by

$$
g_{\Omega}(\tau, u):= \begin{cases}\|u\|_{\Omega}^{2}=\|\nabla u\|_{2, \Omega}^{2}+\lambda\|u\|_{2, \Omega}^{2} & \tau=0, \\ \frac{1}{\tau^{2}}\left\langle I_{\Omega}^{\prime}(\tau u), \tau u\right\rangle & \tau>0 .\end{cases}
$$

Observe that $g_{\Omega}$ is continuous on $\mathbb{R}^{+} \times A_{\Omega}$, moreover, hypotheses (2.1), (2.3) and (2.4) allow to apply Lebesgue Dominate Convergence Theorem to obtain

$$
\lim _{\tau \rightarrow 0^{+}} g_{\Omega}(\tau, u)=\|u\|_{\Omega}^{2}-\lim _{\tau \rightarrow 0^{+}} \int_{\Omega} \frac{f(\tau u) \tau u}{\tau^{2}}=\|u\|_{\Omega}^{2}-\lim _{\tau \rightarrow 0^{+}} \int_{\Omega} \frac{f(\tau u)}{\tau u} u^{2}=\|u\|_{\Omega}^{2} .
$$

So that $g_{\Omega}$ is continuous on $[0,+\infty) \times A_{\Omega}$ and $g_{\Omega}(0, u)>0$. While, (2.1), (2.3) and (2.4) yield

$$
\lim _{\tau \rightarrow+\infty} g_{\Omega}(\tau, u)=\|u\|_{\Omega}^{2}-\lim _{\tau \rightarrow+\infty} \int_{\Omega} \frac{f(\tau u)}{\tau u} u^{2}=\|u\|_{\Omega}^{2}-l_{\infty}\left\|u^{+}\right\|_{2, \Omega}^{2}<0,
$$


where the last inequality is implied by the inequality $\mathcal{G}_{\Omega}(u)<0$. Finally, $(2.3)$ implies that $g_{\Omega}$ is strictly decreasing with respect to $\tau$, so that, since it is a continuous function, there exists a unique $T_{\Omega}(u)>0$ such that $g_{\Omega}\left(T_{\Omega}(u), u\right)=0$, that is

$$
\left\langle I_{\Omega}^{\prime}\left(T_{\Omega}(u) u\right), T_{\Omega}(u) u\right\rangle=0,
$$

i.e. $T_{\Omega}(u) u \in \mathcal{N}_{\Omega}$, showing that $T_{\Omega}$ is well defined.

In order to show that the map $T_{\Omega}$ is continuous in $A_{\Omega}$, let $u$ be in $A_{\Omega}$, so that we can consider $\tilde{\Omega} \subset A$ with $|\tilde{\Omega}|>0$ and $u^{+}(x) \neq 0$ iff $x \in \tilde{\Omega}$ and assume that $\left(u_{n}\right) \in A_{\Omega}$ converges to $u$. The continuity property of $\mathcal{G}_{\Omega}$ implies that there exists $n_{0}$ such that $\mathcal{G}_{\Omega}\left(u_{n}\right)<0$ for every $n \geq n_{0}$, so that there exists $T_{\Omega}\left(u_{n}\right)$ which will be denoted as $T_{n}$. Assume, by contradiction that, up to a subsequence, $T_{n} \rightarrow+\infty$, then (2.1), (2.3) and (2.4) allow to apply Lebesgue dominated convergence Theorem to obtain that

$$
\lim _{n \rightarrow+\infty} \int_{\tilde{\Omega}} \frac{f\left(T_{n} u_{n}\right)}{T_{n} u_{n}} u_{n}^{2}=l_{\infty} \int_{\tilde{\Omega}} u^{2}=l_{\infty} \int_{\Omega}\left(u^{+}\right)^{2} .
$$

Then, by definition of $T_{n}=T_{\Omega}\left(u_{n}\right)$, and using (2.1), (2.3) and (2.4) it results

$$
\begin{aligned}
\|\nabla u\|_{2, \Omega}^{2}+\lambda\|u\|_{2, \Omega}^{2} & =\lim _{n \rightarrow \infty}\left\|\nabla u_{n}\right\|_{2, \Omega}^{2}+\lambda\left\|u_{n}\right\|_{2, \Omega}^{2}=\lim _{n \rightarrow \infty} \int_{\Omega} \frac{f\left(T_{n} u_{n}\right)}{T_{n} u_{n}} u_{n}^{2} \\
& =\lim _{n \rightarrow \infty} \int_{\tilde{\Omega}} \frac{f\left(T_{n} u_{n}\right)}{T_{n} u_{n}} u_{n}^{2}=l_{\infty} \int_{\tilde{\Omega}} u^{2}=l_{\infty} \int_{\Omega}\left(u^{+}\right)^{2}
\end{aligned}
$$

implying $\mathcal{G}_{\Omega}(u)=0$, which is a contradiction. Then, up to a subsequence, $T_{n} \rightarrow T_{0}$; if it were $T_{0}=0$ then (2.1), (2.3) and (2.4) would imply

$$
\|\nabla u\|_{2, \Omega}^{2}+\lambda\|u\|_{2, \Omega}^{2}=\lim _{n \rightarrow \infty}\left\|\nabla u_{n}\right\|_{2, \Omega}^{2}+\lambda\left\|u_{n}\right\|_{2, \Omega}^{2}=\lim _{n \rightarrow \infty} \int_{\Omega} \frac{f\left(T_{n} u_{n}\right)}{T_{n} u_{n}} u_{n}^{2}=0,
$$

again a contradiction, as $\mathcal{G}_{\Omega}(u)<0$.Then, $T_{n} \rightarrow T_{0}>0$ and passing to the limit in (3.13) we derive that $T_{0}=T_{\Omega}(u)$, as $T_{\Omega}(u)$ is unique. In addition, take $\theta \in \mathbb{R}^{N} \backslash\{0\}$ and first observe that $\mathcal{G}_{\Omega}\left(\Phi^{R \theta}\right)=\mathcal{G}\left(\Phi^{R \theta}\right)$, as $\Phi^{R \theta}=0$ for $|x|<\rho$. Moreover, from Lemma 3.4 it results $\mathcal{G}\left(\Phi^{R \theta}\right)=\mathcal{G}(w)+o\left(\varepsilon_{R}\right)$, so that there exists $R_{0}$ such that for every $R \geq R_{0}$, it results $\Phi^{R \theta} \in A \cap A_{\Omega}$. In order to show (3.12), let us consider $\theta \in \mathbb{R}^{N} \backslash\{0\}, R_{n} \rightarrow+\infty$ and set $T_{n}=T_{\Omega}\left(\Phi^{R_{n} \theta}\right)$, which is well defined. By definition, $T_{n}$ satisfies

$$
\left\|\Phi^{R_{n} \theta}\right\|_{\Omega}^{2}=\int_{\Omega} \frac{f\left(T_{n} \Phi^{R_{n} \theta}\right)}{T_{n} \Phi^{R_{n} \theta}}\left(\Phi^{R_{n} \theta}\right)^{2} .
$$

Note that (3.2), (3.9), (2.3), (2.4) and (3.7) imply

$$
\begin{aligned}
\left\|w^{R \theta}\right\|^{2}+o\left(\varepsilon_{R}\right)= & \int_{\mathbb{R}^{N}} \frac{f\left(T_{n} w^{R_{n} \theta}\right)}{T_{n} w^{R_{n} \theta}}\left(w^{R_{n} \theta}\right)^{2}-\int_{B_{2 \rho}(0)} \frac{f\left(T_{n} w^{R_{n} \theta}\right)}{T_{n} w^{R_{n} \theta}}\left(w^{R_{n} \theta}\right)^{2} \\
& +\int_{\{\rho<|x|<2 \rho\}} \frac{f\left(T_{n} \xi w^{R_{n}} \theta\right)}{T_{n} \xi w^{R_{n}} \theta}\left(\xi w^{R_{n}} \theta\right)^{2} \\
= & \int_{\mathbb{R}^{N}} \frac{f\left(T_{n} w^{R_{n} \theta}\right)}{T_{n} w^{R_{n} \theta}}\left(w^{R_{n} \theta}\right)^{2}+o\left(\varepsilon_{R}\right),
\end{aligned}
$$


Moreover, recalling (3.4) and performing a change of variable we obtain

$$
\|w\|^{2}=\int_{\mathbb{R}^{N}} \frac{f\left(T_{n} w\right)}{T_{n} w} w^{2}+o\left(\varepsilon_{R}\right) .
$$

Now, it is easy to see that $\left(T_{n}\right)$ is bounded by contradiction, since if it were not the case then one would obtain that $\mathcal{G}(w)=0$ contradicting the fact that $w \in A$. Then $\left(T_{n}\right)$ has to be bounded, so that we can assume that, up to a subsequence, it converges to $T \in \mathbb{R}$, and $T \neq 0$ arguing again by contradiction. Passing to the limit in (3.14) we get

$$
\|w\|^{2}=\int_{\mathbb{R}^{N}} \frac{f(T w)}{T w} w^{2} .
$$

Then $T=1$ since $w \in \mathcal{N}$.

Remark 3.6. Lemma 3.5 provides the definition of the continuous projection map

$$
\Pi_{\mathcal{N}_{\Omega}}: A_{\Omega} \rightarrow \mathcal{N}_{\Omega}, \quad \text { as } \quad \Pi_{\mathcal{N}_{\Omega}}(u)=T_{\Omega}(u) u .
$$

Up to now, we have obtained asymptotic estimates on a function $\Phi^{R \theta}$. In proving our existence results we will use the following convex combination of $\Phi^{R y}$ and $\Phi^{R x_{0}}$

(3.15) $U_{t}^{R}=t \Phi^{R y}+(1-t) \Phi^{R x_{0}}$, with $x_{0} \in \mathbb{R}^{N},\left|x_{0}\right|=1, y \in \partial B_{2}\left(x_{0}\right), t \in[0,1]$.

As a consequence, we will need also asymptotic informations on quantities involving the functions $\Phi^{R y}$ and $\Phi^{R x_{0}}$ with $y \in \partial B_{2}\left(x_{0}\right)$. Let us start with the following result.

Lemma 3.7. Assume (2.2). Let $x_{0}$ and $y$ be fixed in (3.15). Then it results

$$
\begin{aligned}
\left|\int_{\Omega} \nabla \Phi^{R y} \nabla \Phi^{R x_{0}}+\lambda \Phi^{R y} \Phi^{R x_{0}}\right| \leq & \frac{1}{2} \int_{\mathbb{R}^{N}} f\left(w^{R y}\right) w^{R x_{0}}+\frac{1}{2} \int_{\mathbb{R}^{N}} f\left(w^{R x_{0}}\right) w^{R y} \\
& +o\left(\varepsilon_{R}\right) .
\end{aligned}
$$

Proof. Notice that

$$
\begin{aligned}
\int_{\Omega} \nabla \Phi^{R y} \nabla \Phi^{R x_{0}}+\lambda \Phi^{R y} \Phi^{R x_{0}}= & \int_{\Omega} \nabla \xi\left[w^{R y} \nabla \xi w^{R x_{0}}+\xi\left(w^{R y} \nabla w^{R x_{0}}+\nabla w^{R y} w^{R x_{0}}\right)\right] \\
& +\int_{\mathbb{R}^{N}}\left(\xi^{2}-1\right)\left[\nabla w^{R y} \nabla w^{R x_{0}}+\lambda w^{R y} w^{R x_{0}}\right] \\
& +\int_{\mathbb{R}^{N}} \nabla w^{R y} \nabla w^{R x_{0}}+\lambda w^{R y} w^{R x_{0}} .
\end{aligned}
$$

As $w^{R y}$ and $w^{R x_{0}}$ are solutions of (2.7) we get

$$
\begin{aligned}
\int_{\Omega} \nabla \Phi^{R y} \nabla \Phi^{R x_{0}}+\lambda \Phi^{R y} \Phi^{R x_{0}}=\frac{1}{2} \int_{\mathbb{R}^{N}} f\left(w^{R y}\right) w^{R x_{0}}+\frac{1}{2} \int_{\mathbb{R}^{N}} f\left(w^{R x_{0}}\right) w^{R y} \\
+\int_{\Omega} \nabla \xi\left[w^{R y} \nabla \xi w^{R x_{0}}+\xi\left(w^{R y} \nabla w^{R x_{0}}+\nabla w^{R y} w^{R x_{0}}\right)\right] \\
+\int_{\mathbb{R}^{N}}\left(\xi^{2}-1\right)\left[\nabla w^{R y} \nabla w^{R x_{0}}+\lambda w^{R y} w^{R x_{0}}\right] .
\end{aligned}
$$


Then, the results is proved once one shows that the last two integrals on the right hand side are $o\left(\varepsilon_{R}\right)$. First, we observe that (3.2) imply that there exists a positive constant $C_{0}$ such that

$$
\begin{aligned}
\left|\int_{\Omega} \nabla \xi\left[w^{R y} \nabla \xi w^{R x_{0}}+\xi\left(w^{R y} \nabla w^{R x_{0}}+\nabla w^{R y} w^{R x_{0}}\right)\right]\right| & \leq C_{0} \int_{B_{2 \rho}(0)}\left[\left(w^{R y}\right)^{2}+\left(w^{R x_{0}}\right)^{2}\right] \\
& +C_{0} \int_{B_{2 \rho}(0)}\left[\left|\nabla w^{R y}\right|^{2}+\left|\nabla w^{R x_{0}}\right|^{2}\right] \\
& \leq o\left(\varepsilon_{R}\right)
\end{aligned}
$$

where the last inequality is implied by (3.7). In addition exploiting again (3.2) and (3.7) one obtains

$$
\begin{aligned}
\left|\int_{\mathbb{R}^{N}}\left(\xi^{2}-1\right)\left[\nabla w^{R y} \nabla w^{R x_{0}}+\lambda w^{R y} w^{R x_{0}}\right]\right| & \leq \frac{1}{2} \int_{B_{2 \rho}(0)}\left[\left|\nabla w^{R y}\right|^{2}+\left|\nabla w^{R x_{0}}\right|^{2}\right] \\
+\frac{\lambda}{2} \int_{B_{2 \rho}(0)}\left[\left(w^{R y}\right)^{2}+\left(w^{R x_{0}}\right)^{2}\right] & \leq o\left(\varepsilon_{R}\right)
\end{aligned}
$$

yielding the conclusion.

In the following lemma we prove some crucial estimates for the map $T_{\Omega}\left(U_{t}^{R}\right)$.

Lemma 3.8. Assume conditions (2.1), (2.2), (2.3), (2.4) and let $x_{0}, y$ and t be given in (3.15). Then, there exists $R_{1}$ such that the following conclusions hold:

i) $U_{t}^{R} \in A_{\Omega}$, for every $R \geq R_{1}$;

ii) there exists a positive constant $L$ such that

$$
\left|T_{\Omega}\left(U_{t}^{R}\right)\right| \leq L, \quad \text { for every }(t, R) \in[0,1] \times\left[R_{1},+\infty\right) .
$$

iii) For every $t_{0} \in(0,1)$, it holds

$$
\lim _{(t, R) \rightarrow\left(t_{0},+\infty\right)} T_{\Omega}\left(U_{t}^{R}\right)=\frac{1}{t_{0}} \text { if and only if } t_{0}=\frac{1}{2} .
$$

Proof. First of all, let us note that (3.9) and Lemmas 3.2 and 3.7 imply that the following inequality holds for every $y \in \partial B_{2}\left(x_{0}\right)$ (recall (3.15))

$$
\begin{aligned}
\left\|U_{t}^{R}\right\|_{\Omega}^{2} & =\left\|U_{t}^{R}\right\|^{2}=t^{2}\left\|\Phi^{R y}\right\|^{2}+(1-t)^{2}\left\|\Phi^{R x_{0}}\right\|^{2} \\
& +2 t(1-t) \int_{\mathbb{R}^{N}} \nabla \Phi^{R y} \nabla \Phi^{R x_{0}}+\Phi^{R y} \Phi^{R x_{0}} \\
& \leq t^{2}\left\|w^{R y}\right\|+(1-t)^{2}\left\|w^{R x_{0}}\right\|^{2}+2 t(1-t) \varepsilon_{R}+o\left(\varepsilon_{R}\right) .
\end{aligned}
$$

Then, recalling the definition of $\mathcal{G}_{\Omega}$ stated in Lemma 3.5, using again (3.9), and applying Lemma 3.7, one obtains

$$
\begin{aligned}
\mathcal{G}_{\Omega}\left(U_{t}^{R}\right) \leq & t^{2}\left\|w^{R y}\right\|^{2}+(1-t)^{2}\left\|w^{R x_{0}}\right\|^{2}-l_{\infty} t^{2}\left\|w^{R y}\right\|^{2}-l_{\infty}(1-t)^{2}\left\|w^{R x_{0}}\right\|^{2} \\
& +2 t(1-t) \varepsilon_{R}+2 l_{\infty} t(1-t) \int_{\mathbb{R}^{N}} w^{R y} w^{R x_{0}}+o\left(\varepsilon_{R}\right) \\
= & t^{2} \mathcal{G}(w)+(1-t)^{2} \mathcal{G}(w)+2 t(1-t) \varepsilon_{R} \\
& +2 l_{\infty} t(1-t) \int_{\mathbb{R}^{N}} w^{R y} w^{R x_{0}}+o\left(\varepsilon_{R}\right) .
\end{aligned}
$$


Thanks to (3.3) we can apply Lemma 2.1 in [1] with $\bar{\mu}=\sqrt{\lambda} / 2$ to deduce

$$
\lim _{R \rightarrow+\infty} \int_{\mathbb{R}^{N}} w^{R y} w^{R x_{0}} \leq \lim _{R \rightarrow+\infty} e^{-\sqrt{\lambda} R}=0 .
$$

Then, as $\mathcal{G}(w)<0$, there exists $R_{1}$ such that for every $R>R_{1}$ it holds $\mathcal{G}_{\Omega}\left(U_{t}^{R}\right)<0$, for every $y \in \partial B_{2}\left(x_{0}\right)$, yielding conclusion $i$ ). In order to prove conclusion $i$ ), first note that $T_{\Omega}\left(U_{t}^{R}\right)$ is well defined; then, we argue by contradiction and suppose that there exist sequences $R_{n} \rightarrow+\infty, t_{n} \in[0,1]$ and $y_{n} \in \partial B_{2}\left(x_{0}\right)$ such that

$$
U_{n}:=t_{n} \Phi^{R_{n} y_{n}}+\left(1-t_{n}\right) \Phi^{R_{n} x_{0}} \text { satisfies } T_{n}:=T_{\Omega}\left(U_{n}\right) \rightarrow+\infty .
$$

Since $t_{n} \in[0,1]$ we can suppose, up to a subsequence, that there exists $t_{0} \in[0,1]$ such that $t_{n} \rightarrow t_{0}$. Three cases may occur: either $t_{0}=0$, or $t_{0}=1$ or $t_{0} \in(0,1)$. Suppose first that $t_{0}=0$, and observe that $T_{n} U_{n} \in \mathcal{N}_{\Omega}$ so that

$$
\left\|U_{n}\right\|_{\Omega}^{2}=\int_{\Omega} \frac{f\left(T_{n} U_{n}\right)}{T_{n} U_{n}} U_{n}^{2} .
$$

Then, taking into account that $t_{n} \rightarrow 0, R_{n} \rightarrow+\infty$ and exploiting (3.18), we obtain

$$
\lim _{n \rightarrow+\infty}\left\|U_{n}\right\|_{\Omega}^{2}=\|w\|^{2} .
$$

With respect to the right hand side of (3.20) we observe that the property of the function $\xi$, joint with assumptions (2.3) and (2.4) give that there exists a positive constant $C_{1}$ such that

$$
\int_{\Omega} \frac{f\left(T_{n} U_{n}\right)}{T_{n} U_{n}}\left[t_{n}^{2}\left(\Phi^{R_{n} y_{n}}\right)^{2}+2 t_{n}\left(1-t_{n}\right) \Phi^{R_{n} y_{n}} \Phi^{R_{n} x_{0}}\right] \leq C_{1} t_{n}\|w\|_{2}^{2}
$$

Moreover, one observes that

$$
\int_{\Omega} \frac{f\left(T_{n} U_{n}\right)}{T_{n} U_{n}}\left(\Phi^{R_{n} x_{0}}\right)^{2}=\int_{\Omega} \frac{f\left(T_{n} U_{n}\right)}{T_{n} U_{n}}\left(w^{R_{n} x_{0}}\right)^{2}+\int_{\Omega} \frac{f\left(T_{n} U_{n}\right)}{T_{n} U_{n}}\left[\left(\Phi^{R_{n} x_{0}}\right)^{2}-\left(w^{R_{n} x_{0}}\right)^{2}\right]
$$

and assumptions (2.3), (2.4) and (3.9) yield

$$
\int_{\Omega} \frac{f\left(T_{n} U_{n}\right)}{T_{n} U_{n}}\left[\left(\Phi^{R_{n} x_{0}}\right)^{2}-\left(w^{R_{n} x_{0}}\right)^{2}\right] \leq 2 l_{\infty} \int_{B_{2 \rho}(0)}\left(w^{R_{n} x_{0}}\right)^{2} \leq o\left(\varepsilon_{R_{n}}\right) .
$$

In addition, since

$$
\begin{array}{r}
\int_{\Omega} \frac{f\left(T_{n} U_{n}\right)}{T_{n} U_{n}}\left(w^{R_{n} x_{0}}\right)^{2}=\int_{\mathbb{R}^{N}} \frac{f\left(T_{n}\left(t_{n} \xi w^{R_{n} y_{n}}+\left(1-t_{n}\right) \xi w^{R_{n} x_{0}}\right)\right)}{T_{n}\left(t_{n} \xi w^{R_{n} y_{n}}+\left(1-t_{n}\right) \xi w^{R_{n} x_{0}}\right)}\left(w^{R_{n} x_{0}}\right)^{2} \\
=\int_{\mathbb{R}^{N}} \frac{f\left(T_{n}\left(t_{n} \xi^{-R_{n} x_{0}} w^{-R_{n}\left(y_{n}+x_{0}\right)}+\left(1-t_{n}\right) \xi^{-R_{n} x_{0}} w\right)\right)}{T_{n}\left(t_{n} \xi^{-R_{n} x_{0}} w^{-R_{n}\left(y_{n}+x_{0}\right)}+\left(1-t_{n}\right) \xi^{-R_{n} x_{0}} w\right)} w^{2}
\end{array}
$$

and as (3.2) and (3.19) imply

$$
T_{n}\left(t_{n} \xi^{-R_{n} x_{0}} w^{-R_{n}\left(y_{n}+x_{0}\right)}+\left(1-t_{n}\right) \xi^{-R_{n} x_{0}} w\right) \geq T_{n}\left(1-t_{n}\right) \xi\left(x+R_{n} x_{0}\right) w(x) \rightarrow+\infty
$$

almost everywhere in $\mathbb{R}^{N}$, (2.4) and (2.3) yields

$$
\lim _{n \rightarrow+\infty} \int_{\Omega} \frac{f\left(T_{n} U_{n}\right)}{T_{n} U_{n}}\left(w^{R_{n} x_{0}}\right)^{2}=l_{\infty} \int_{\mathbb{R}^{N}} w^{2} .
$$

Then (3.21), (3.22), (3.23), (3.25) allow us to pass to the list in (3.20) to obtain $\mathcal{G}(w)=0$, which is a contradiction as $w \in A$ (defined in Lemma 3.5). 
The case in which $t_{0}=1$ is similar to the case $t_{0}=0$ by exchanging the role of $t_{n}$ with the one of $1-t_{n}$. Let us handle the third case and suppose that $t_{0} \in(0,1)$ and come back to (3.18) to observe that (3.9) and Lemma 3.7 imply that

$$
\lim _{n \rightarrow+\infty}\left\|U_{n}\right\|_{\Omega}^{2}=\left[t_{0}^{2}+\left(1-t_{0}\right)^{2}\right]\|w\|^{2} .
$$

In order to study the right hand side of (3.20) notice that, by (2.3) and (3.2)

$$
0 \leq \limsup _{n \rightarrow+\infty} \int_{\Omega} \frac{f\left(T_{n} U_{n}\right)}{T_{n} U_{n}} \Phi^{R_{n} y_{n}} \Phi^{R_{n} x_{0}} \leq l_{\infty} \lim _{n \rightarrow+\infty} \int_{\mathbb{R}^{N}} w(x) w\left(x-R_{n}\left(x_{0}-y\right)\right)=0
$$

and as $t_{0} \in(0,1)$ it results

$$
\lim _{n \rightarrow+\infty} 2 t_{n}\left(1-t_{n}\right) \int_{\Omega} \frac{f\left(T_{n} U_{n}\right)}{T_{n} U_{n}} \Phi^{R_{n} y_{n}} \Phi^{R_{n} x_{0}}=0 .
$$

Then, taking into account that (3.23) holds also for $y$ in the place of $x_{0}$, one obtains

$$
\begin{aligned}
\lim _{n \rightarrow+\infty} \int_{\Omega} \frac{f\left(T_{n} U_{n}\right)}{T_{n} U_{n}} U_{n}^{2}= & \lim _{n \rightarrow+\infty} t_{n}^{2} \int_{\Omega} \frac{f\left(T_{n} U_{n}\right)}{T_{n} U_{n}}\left(w^{R_{n} y_{n}}\right)^{2} \\
& +\lim _{n \rightarrow+\infty}\left(1-t_{n}\right)^{2} \int_{\Omega} \frac{f\left(T_{n} U_{n}\right)}{T_{n} U_{n}}\left(w^{R_{n} x_{0}}\right)^{2} .
\end{aligned}
$$

Arguing as in (3.24) in the above integrals separately, exploiting (3.2) and (3.19) and taking into account that $t_{0} \in(0,1)$ one deduces

$$
T_{n}\left[t_{n} \xi^{-R_{n} y_{n}} w+\left(1-t_{n}\right) \xi^{-R_{n} y_{n}} w^{R_{n}\left(x_{0}-y_{n}\right)}\right] \geq T_{n} t_{n} \xi\left(x-R_{n} y_{n}\right) w \rightarrow+\infty \quad \text { a.e. in } \Omega \text {. }
$$

Therefore, we can use (2.4) and (2.3) to apply Lebesgue dominated convergence Theorem and obtain

$$
\lim _{n \rightarrow+\infty} \int_{\Omega} \frac{f\left(T_{n} U_{n}\right)}{T_{n} U_{n}} U_{n}^{2}=\left[t_{0}^{2}+\left(1-t_{0}\right)^{2}\right] l_{\infty}\|w\|_{2}^{2} .
$$

Finally, passing to the limit in (3.20) and using (3.26), (3.27) it follows

$$
\left[t_{0}^{2}+\left(1-t_{0}\right)^{2}\right]\|w\|^{2}=\left[t_{0}^{2}+\left(1-t_{0}\right)^{2}\right] l_{\infty}\|w\|_{2}^{2},
$$

which again contradicts the fact that $w \in A$, yielding (3.16). In order to prove (3.17), let us first show that

$$
\lim _{(t, R) \rightarrow\left(\frac{1}{2},+\infty\right)} T_{\Omega}\left(U_{t}^{R}\right)=2 .
$$

Arguing again by contradiction and supposing that there exist $\delta>0$ and subsequences $R_{k} \rightarrow+\infty, t_{k} \rightarrow 1 / 2$ and $y_{k} \in \partial B_{2}\left(x_{0}\right)$ such that, the sequence

$$
T_{k}:=T_{\Omega}\left(t_{k} \Phi^{R_{k} y_{k}}+\left(1-t_{k}\right) \Phi^{R_{k} x_{0}}\right) \text { satisfies }\left|T_{k}-2\right| \geq \delta .
$$

Estimate (3.16) implies that there exists $T$ such that, up to a subsequence, $T_{k} \rightarrow T$. Then (3.20) becomes

$$
\left\|U_{k}\right\|_{\Omega}^{2}=\int_{\Omega} \frac{f\left(T_{k} U_{k}\right)}{T_{k} U_{k}} U_{k}^{2}
$$


and (3.26) allows to take the limit on the left hand side; on the other hand, we can argue in analogous way as in the case $t_{0} \in(0,1)$ to obtain

$$
\frac{1}{2}\|w\|^{2}=\int_{\mathbb{R}^{N}} \frac{f\left(\frac{T}{2} w\right)}{\frac{T}{2} w} \frac{w^{2}}{2} .
$$

As $w$ is a solution of the limit problem (2.7) this implies

$$
\int_{\mathbb{R}^{N}}\left[\frac{f(w)}{w}-\frac{f\left(\frac{T}{2} w\right)}{\frac{T}{2} w}\right] w^{2}=0,
$$

yielding (3.29) thanks to (2.3). On the other hand, suppose that there exists $t_{0} \in$ $(0,1)$ such that the limit in (3.17) holds and use (3.9) and Lemma (3.7) to take the limit in (3.30) and obtain

$$
t_{0}^{2}\|w\|^{2}+\left(1-t_{0}\right)^{2}\|w\|^{2}=t_{0}^{2} \int_{\mathbb{R}^{N}} \frac{f(w)}{w} w^{2}+\int_{\mathbb{R}^{N}} \frac{f\left(w\left(1-t_{0}\right) / t_{0}\right)}{w\left(1-t_{0}\right) / t_{0}}\left(1-t_{0}\right)^{2} w^{2}
$$

that is

$$
0=\left(1-t_{0}\right)^{2} \int_{\mathbb{R}^{N}}\left[\frac{f(w)}{w}-\frac{f\left(w\left(1-t_{0}\right) / t_{0}\right)}{w\left(1-t_{0}\right) / t_{0}}\right] w^{2} .
$$

Finally, (2.3) implies that $t_{0}=1 / 2$ yielding the conclusion.

Lemma 3.9. Assume condition (2.2) and let $x_{0}, y$ and $t$ be given in (3.15). Then, it results

$$
\begin{gathered}
\mid \int_{\mathbb{R}^{N}}\left[F\left(T_{R}^{t} U_{t}^{R}\right)-F\left(T_{R}^{t} t \Phi^{R y}\right)-F\left(T_{R}^{t}(1-t) \Phi^{R x_{0}}\right)\right] \\
+\int_{\mathbb{R}^{N}}\left[-f\left(T_{R}^{t} t \Phi^{R y}\right) T_{R}^{t}(1-t) \Phi^{R x_{0}}-f\left(T_{R}^{t}(1-t) \Phi^{R x_{0}}\right) T_{t}^{R} t \Phi^{R y}\right] \mid \leq o\left(\varepsilon_{R}\right),
\end{gathered}
$$

where $T_{t}^{R}=T_{\Omega}\left(U_{t}^{R}\right)$.

Proof. We use Lemma 2.2 in [1], with $\alpha:=\min \left\{\left(p_{1}+1\right) / 4,1\right\}$ and $p_{1}$ as in (2.2), and we take into account (3.2) and (3.16) to obtain that

$$
\begin{array}{r}
\mid \int_{\mathbb{R}^{N}}\left[F\left(T_{R}^{t} U_{t}^{R}\right)-F\left(T_{R}^{t} t \Phi^{R y}\right)-F\left(T_{R}^{t}(1-t) \Phi^{R x_{0}}\right)\right] \\
+\int_{\mathbb{R}^{N}}\left[-f\left(T_{R}^{t} t \Phi^{R y}\right) T_{R}^{t}(1-t) \Phi^{R x_{0}}-f\left(T_{R}^{t}(1-t) \Phi^{R x_{0}}\right) T_{R}^{t} t \Phi^{R y}\right] \mid \\
\leq\left[T_{R}^{t} t\right]^{2 \alpha}\left[T_{R}^{t}(1-t)\right]^{2 \alpha} \int_{\mathbb{R}^{N}}\left|\Phi^{R y}\right|^{2 \alpha}\left|\Phi^{R x_{0}}\right|^{2 \alpha} \\
\leq L^{4 \alpha} \int_{\mathbb{R}^{N}} w^{2 \alpha}(x) w^{2 \alpha}\left(x-R\left(y-x_{0}\right)\right) d x .
\end{array}
$$

Since $2 \alpha>1$, we have that, denoting with $\varphi(x)=w^{2 \alpha}(x)$, the first hypothesis in Lemma 3.1 is satisfied with $c=0$. Then Lemma 3.1 implies that

$$
\lim _{R \rightarrow \infty}(2 R)^{(N-1) / 2} e^{2 R \sqrt{\lambda}} \int_{\mathbb{R}^{N}} w^{2 \alpha}(x) w^{2 \alpha}\left(x-R\left(y-x_{0}\right)\right) d x=0 .
$$

Then, Lemma 3.2 yields the conclusion. 
Lemma 3.10. Assume (2.1), (2.2), (2.3) and let $x_{0}$ and $y$ be given in (3.15). Then, There exist $C=C\left(\|w\|_{\infty}\right)>0$ such that, for all $\tau_{1}, \tau_{2} \in[0,+\infty)$, it results

$$
\int_{\mathbb{R}^{N}} f\left(\tau_{1} w^{R x_{0}}\right) \tau_{2} w^{R y} \geq \min \left\{\tau_{1}, \tau_{2}\right\} O\left(\varepsilon_{R}\right), \int_{\mathbb{R}^{N}} f\left(\tau_{1} w^{R y}\right) \tau_{2} w^{R x_{0}} \geq \min \left\{\tau_{1}, \tau_{2}\right\} O\left(\varepsilon_{R}\right) .
$$

Proof. As, performing a change of variable we have

$$
\begin{aligned}
& \int_{\mathbb{R}^{N}} f\left(\tau_{1} w^{R x_{0}}\right) \tau_{2} w^{R y}=\int_{\mathbb{R}^{N}} f\left(\tau_{1} w\right) \tau_{2} w^{R\left(x_{0}-y\right)}, \\
& \int_{\mathbb{R}^{N}} f\left(\tau_{1} w^{R y}\right) \tau_{2} w^{R x_{0}}=\int_{\mathbb{R}^{N}} f\left(\tau_{1} w\right) \tau_{2} w^{R\left(y-x_{0}\right)},
\end{aligned}
$$

it is enough to show the first inequality, since the other will follow by a similar argument. In order to do this, taking into account Lemma 3.2, it is sufficient to show that there exists a constant $C$ such that

$$
\int_{\mathbb{R}^{N}} f\left(\tau_{1} w^{R x_{0}}\right) \tau_{2} w^{R y} \geq C R^{-(N-1) / 2} e^{-2 R \sqrt{\lambda}} .
$$

Note that, the positive minimum of $w(x)$ in the ball $B_{1}(0)$ is achieved for $|x|=1$; let us denote this minimum value as $\alpha_{0}>0$. As a consequence of hypothesis (2.3) we have that the function $g(u)=f(u) / u$ is continuous and monotone increasing in the interval $\left[\alpha_{0},\|w\|_{\infty}\right]$, so that $g(u) \geq C:=g\left(\alpha_{0}\right)$. Then we obtain

$$
\begin{aligned}
\int_{\mathbb{R}^{N}} f\left(\tau_{1} w^{R x_{0}}\right) \tau_{2} w^{R y} & \geq \tau_{1} \tau_{2} \int_{B_{1}(0)} \frac{f\left(\tau_{1} w\right)}{\tau_{1} w} w w^{R\left(x_{0}-y\right)} \\
& \geq \tau_{1} \tau_{2} C \int_{B_{1}(0)} w(x) w\left(x-R\left(x_{0}-y\right)\right) d x
\end{aligned}
$$

Note that, for every $x \in B_{1}(0)$ it results for every $R \geq 1$

$$
2 R-1 \leq\left|R\left(y-x_{0}\right)\right|-|x| \leq\left|x-R\left(y-x_{0}\right)\right| \leq 2 R+1 \leq 3 R .
$$

In the following we will denote with $C$ possibly different positive constants. Condition (3.3) implies that for sufficiently large $R$ there exists a constant $C$ such that

$$
w\left(x-R\left(x_{0}-y\right)\right) \geq C\left|x-R\left(y-x_{0}\right)\right|^{-(N-1) / 2} e^{-\sqrt{\lambda}\left|x-R\left(y-x_{0}\right)\right|}
$$

and (3.32) gives

$$
w\left(x-R\left(x_{0}-y\right)\right) \geq C R^{-(N-1) / 2} e^{-2 R \sqrt{\lambda}} .
$$

Using this inequality in (3.31) and applying Lemma 3.2 yield the conclusion.

Lemma 3.11. Assume (2.1), (2.2), (2.3), and let $x_{0}$ and $y$ be given in (3.15). Then, for every $\tau \in[0,+\infty)$, it results

$$
\begin{aligned}
&\left|\int_{\mathbb{R}^{N}} f\left(\tau \Phi^{R y}\right) \Phi^{R x_{0}}-f\left(\tau w^{R y}\right) w^{R x_{0}}\right| \leq 2 D \max \left\{\tau^{p_{1}}, \tau^{p_{2}}\right\} o\left(\varepsilon_{R}\right), \\
&\left|\int_{\mathbb{R}^{N}} \tau f\left(w^{R x_{0}}\right) w^{R y}-f\left(\tau w^{R x_{0}}\right) w^{R y}\right| \leq|\tau-1|\left[1+D \tau^{\max \left\{p_{1}, p_{2}\right\}-1}\right] O\left(\varepsilon_{R}\right) .
\end{aligned}
$$


Proof. The first inequality is a direct consequence of (2.3), (2.2), (3.2) and (3.7). Indeed it results

$$
\begin{gathered}
\int_{\mathbb{R}^{N}}\left|f\left(\tau \Phi^{R y}\right) \Phi^{R x_{0}}-f\left(\tau w^{R y}\right) w^{R x_{0}}\right| \leq 2 \int_{B_{2 \rho}(0)} f\left(\tau w^{R y}\right) w^{R x_{0}} \\
\leq 2 D \max \left\{\tau^{p_{1}}, \tau^{p_{2}}\right\} \int_{B_{2 \rho}(0)}\left[\left(w^{R y}\right)^{p_{1}}+\left(w^{R y}\right)^{p_{2}}\right] w^{R x_{0}} \leq 2 D \max \left\{\tau^{p_{1}}, \tau^{p_{2}}\right\} o\left(\varepsilon_{R}\right) .
\end{gathered}
$$

In order to show (3.34), consider the function $g(\tau):=\tau f\left(w^{R x_{0}}\right)-f\left(\tau w^{R x_{0}}\right)$, and suppose, without loss of generality, that $\tau>1$. The mean value theorem implies that there exists $\theta \in(1, \tau)$ such that

$$
g(\tau)=g(\tau)-g(1)=g^{\prime}(\theta)(\tau-1)=\left[f\left(w^{R x_{0}}\right)-f^{\prime}\left(\theta w^{R x_{0}}\right) w^{R x_{0}}\right](\tau-1) .
$$

Substituting in the integral in (3.34) we obtain

$$
\begin{aligned}
\left|\int_{\mathbb{R}^{N}}\left[\tau f\left(w^{R x_{0}}\right)-f\left(\tau w^{R x_{0}}\right)\right] w^{R y}\right| & =|\tau-1|\left|\int_{\mathbb{R}^{N}}\left[f\left(w^{R x_{0}}\right)-f^{\prime}\left(\theta w^{R x_{0}}\right) w^{R x_{0}}\right] w^{R y}\right| \\
& \leq|\tau-1| \int_{\mathbb{R}^{N}} f\left(w^{R x_{0}}\right) w^{R y}+\left|f^{\prime}\left(\theta w^{R x_{0}}\right)\right| w^{R x_{0}} w^{R y} .
\end{aligned}
$$

Applying Lemma 3.2, hypothesis (2.2) ad taking into account that $\theta \in(1, \tau)$ one obtains

$$
\begin{aligned}
& \left|\int_{\mathbb{R}^{N}}\left[\tau f\left(w^{R x_{0}}\right)-f\left(\tau w^{R x_{0}}\right)\right] w^{R y}\right| \leq|\tau-1| \varepsilon_{R} \\
& \quad+D|\tau-1| \tau^{\max \left\{p_{1}, p_{2}\right\}-1} \int_{\mathbb{R}^{N}}\left[\left(w^{R x_{0}}\right)^{p_{1}} w^{R y}+\left(w^{R x_{0}}\right)^{p_{2}} w^{R y}\right] .
\end{aligned}
$$

Applying Lemma 3.1 with $\varphi_{1}=w$ and $\varphi_{2}=w^{p_{1}}$ (see also the argument of the proof of Lemma 3.2), we obtain that

$$
\int_{\mathbb{R}^{N}}\left[\left(w^{R x_{0}}\right)^{p_{1}} w^{R y}+\left(w^{R x_{0}}\right)^{p_{2}} w^{R y}\right] \leq O\left(\varepsilon_{R}\right)
$$

so that the conclusion follows.

\section{Compactness Condition}

In this section we will find the level interval where Palais-Smale condition holds.

Lemma 4.1. Assume (2.1), (2.3),(2.4). There holds

$$
m_{\Omega}=m
$$

and $m_{\Omega}$ is not attained.

Proof. Since any $u \in H_{0}^{1}(\Omega)$ can be extended as zero outside $\Omega$, we may consider $H_{0}^{1}(\Omega) \subset H^{1}\left(\mathbb{R}^{N}\right)$ and so $m_{\Omega} \geq m$. On the other hand, applying Lemma 3.5 to the sequence $\phi_{n}:=\Phi^{R_{n} \theta}$, with $R_{n} \rightarrow+\infty$, it follows that there exists $n_{0}$ such that for every $n \geq n_{0}$ there exists $T_{n}>0$ such that $T_{n}\left(\phi_{n}\right) \phi_{n} \in \mathcal{N}_{\Omega}$. Then, taking into account (1.5), (3.10) and (3.12) yield

$$
m_{\Omega} \leq I_{\Omega}\left(T_{n}\left(\phi_{n}\right) \phi_{n}\right)=I_{\Omega}\left(T_{n}\left(\Phi^{R_{n} \theta}\right) \Phi^{R_{n} \theta}\right) \rightarrow I(w)=m .
$$


Finally, assume that there exists $u \in H_{0}^{1}(\Omega)$ such that $I_{\Omega}(u)=m_{\Omega}$. Then $\bar{u}$, extension by zero of $u$ outside $\Omega$, would be a minimizer of the problem at infinity not positive on the whole $\mathbb{R}^{N}$ contradicting the maximum principle.

The remaining of this section is devoted to recover compactness properties for $I_{\Omega}$. The main difficulty in our context is due to the fact that $f$ is asymptotically linear. Moreover, we will need a compactness property just for sequences belonging to the Nehari manifold $\mathcal{N}_{\Omega}$, then we first prove the following result.

Lemma 4.2. Assume (2.1), (2.2), (2.3), (2.4), (2.5), (2.6). Let $d \in \mathbb{R}^{+}$and $\left(u_{n}\right)$ be such that

$$
\left(u_{n}\right) \in \mathcal{N}_{\Omega} \quad \text { and } \quad \lim _{n \rightarrow+\infty} I_{\Omega}\left(u_{n}\right)=d>0
$$

then $\left(u_{n}\right)$ is bounded.

Proof. The proof can be started as in Proposition 3.20 in [28] and it can be concluded as in Lemma 5.3 in [27]. We give here a brief summary. First, note that as $u_{n} \in \mathcal{N}_{\Omega}$ it results

$$
I_{\Omega}\left(p u_{n}\right)=\frac{p^{2}}{2}\left\|u_{n}\right\|_{\Omega}^{2}-\int_{\Omega} F\left(p u_{n}\right) d x=\int_{\Omega} \frac{p^{2}}{2} f\left(u_{n}\right) u_{n}-F\left(p u_{n}\right) .
$$

For every $v \in \mathcal{N}_{\Omega}$, let us consider the one-variable function $h: \mathbb{R}^{+} \rightarrow \mathbb{R}$

$$
h_{v}(p):=\frac{p^{2}}{2} f(v) v-F(p v), \quad I(p v)=\int_{\Omega} h_{v}(p) .
$$

Notice that (4.1) implies that there exists a positive constant $C=C(d)$ such that

$$
\left|I_{\Omega}\left(u_{n}\right)\right| \leq C
$$

so that, condition (2.3) implies that, for every fixed $x \in \mathbb{R}^{N}, h_{u_{n}}(p)$ has a unique maximum point for $p=1$ so that $I_{\Omega}\left(p u_{n}\right) \leq I_{\Omega}\left(u_{n}\right) \leq C$. Recalling (4.2), one obtains

$$
\int_{\Omega} F\left(p u_{n}\right) \geq \frac{p^{2}}{2}\left\|u_{n}\right\|^{2}-C
$$

Then, assuming by contradiction that, up to a subsequence, $\left\|u_{n}\right\|_{\Omega} \rightarrow+\infty$ and setting $v_{n}=p_{n} u_{n}$ with $p_{n}=2 \sqrt{C} /\left\|u_{n}\right\|_{\Omega}$, we obtain the uniform lower bound

$$
\int_{\Omega} F\left(v_{n}\right) \geq C \text {. }
$$

This lower bound, hypothesis (2.2) and Lions Lemma (see [25]) imply that there exist positive numbers $r$ and $\delta$ and a sequence $\left(y_{n}\right) \in \mathbb{R}^{N}$ such that

$$
\liminf _{n \rightarrow \infty} \int_{B_{r}\left(y_{n}\right)} v_{n}^{2} d x \geq \delta .
$$

Then, we have to handle two different possible situations: either $y_{n}$ is bounded, or, up to a subsequence, $\left|y_{n}\right| \rightarrow+\infty$ as $n \rightarrow+\infty$. 
In the first case we deduce from (4.3) and recalling that $v_{n} \equiv 0$ outside $\Omega$, that there exists $r_{1}>\rho$ such that

$$
\liminf _{n \rightarrow \infty} \int_{B_{r_{1}}(0)} v_{n}^{2} d x \geq \frac{\delta}{2}
$$

and the same lower bound holds for the weak limit $v$ of $v_{n}$ (up to a subsequence). Then, as $v_{n} \equiv 0$ in $\mathbb{R}^{N} \backslash \Omega$ there exists a subset $\Lambda \in B_{r_{1}}(0) \cap \Omega$ with positive measure and such that $v(x)>0$ in $\Lambda$, so as $u_{n}(x)=2 \sqrt{C} v_{n}(x)\left\|u_{n}\right\|_{\Omega}$, it follows that $u_{n}(x) \rightarrow+\infty$ for all $x \in \Lambda$. This immediately leads to a contradiction using (2.1) (2.5) and (4.1) (for more details see Lemma 5.3 in [27]).

Then $\left(y_{n}\right)$ cannot be bounded, and, up to a subsequence, we obtain $\left|y_{n}\right| \rightarrow+\infty$; moreover, we can assume that $B_{r}\left(y_{n}\right) \subset \Omega$ for $n$ sufficiently large. Then, it is possible to argue analogously on the sequence $\tilde{v}_{n}=v_{n}\left(x+y_{n}\right)$, obtaining, as before, a set $\Lambda \subseteq B_{r}(0)$ with positive measure such that $u_{n}\left(x+y_{n}\right) \rightarrow+\infty$ for every $x \in \Lambda$. The contradiction follows again by using (2.5) (see Lemma 5.3 in [27]). Indeed, (4.1) yields

$$
\begin{aligned}
C & \geq \limsup _{n \rightarrow+\infty} I_{\Omega}\left(u_{n}\right)-\left\langle I_{\Omega}^{\prime}\left(u_{n}\right), u_{n}\right\rangle \geq \int_{B_{r}\left(y_{n}\right)} \frac{1}{2} f\left(u_{n}\right) u_{n}-F\left(u_{n}\right) \\
& =\int_{B_{r}(0)} \frac{1}{2} f\left(u_{n}\left(x+y_{n}\right)\right) u_{n}\left(x+y_{n}\right)-F\left(u_{n}\left(x+y_{n}\right)\right) d x \\
& \geq \int_{\Lambda} \frac{1}{2} f\left(u_{n}\left(x+y_{n}\right)\right) u_{n}\left(x+y_{n}\right)-F\left(u_{n}\left(x+y_{n}\right)\right) d x
\end{aligned}
$$

and the last integral goes to plus infinity thanks to (2.5). Then, we reach a contradiction, proving the Lemma.

Remark 4.3. Notice that in order to prove that $\left(u_{n}\right)$ is bounded the classical information $I_{\Omega}^{\prime}\left(u_{n}\right) \rightarrow 0$ can be substituted by the information $\left(u_{n}\right) \subseteq \mathcal{N}_{\Omega}$. Moreover, note that, in order to prove Lemma 4.2, it is sufficient to assume (2.2) for $k=0$.

In the following lemma we will show that $\left(u_{n}\right)$ is a Palais-Smale sequence in the whole space.

Lemma 4.4. Assume (2.1), (2.2), (2.3), (2.4), (2.5), (2.6). Let ( $\left.u_{n}\right)$ satisfy (4.1) and be such that

$$
\nabla_{\mathcal{N}_{\Omega}} I_{\Omega}\left(u_{n}\right) \rightarrow 0
$$

Then $I_{\Omega}^{\prime}\left(u_{n}\right) \rightarrow 0$ in $H^{-1}(\Omega)$.

Proof. First of all, from (4.4), recalling (1.4), we obtain a sequence $\left(\mu_{n}\right) \subseteq \mathbb{R}$ such that

$$
I_{\Omega}^{\prime}\left(u_{n}\right)-\mu_{n} N_{\Omega}^{\prime}\left(u_{n}\right) \rightarrow 0,
$$

where $N_{\Omega}$ is defined in Remark 2.5. Moreover, we can use Lemma 4.2 to obtain that $\left(u_{n}\right)$ is bounded, so that there exists $u \in H_{0}^{1}(\Omega)$ such that, up to a subsequence, $u_{n} \rightarrow u$ weakly in $H_{0}^{1}(\Omega)$ and $u_{n} \rightarrow u$ almost everywhere. Let us first show that $\left(\mu_{n}\right)$ is bounded arguing by contradiction, so that we assume that, up to a subsequence, 
$\left|\mu_{n}\right| \rightarrow+\infty$ and set $t_{n}=\mu_{n} /\left|\mu_{n}\right|$. Since $\left|t_{n}\right|=1$ there exists $t_{0}$ such that, up to a subsequence, $\left|t_{0}\right|=1$ and $t_{n} \rightarrow t_{0}$ in $\mathbb{R}$. Moreover, it results

$$
\frac{1}{\left|\mu_{n}\right|} I_{\Omega}^{\prime}\left(u_{n}\right)-t_{n} N_{\Omega}^{\prime}\left(u_{n}\right) \rightarrow 0
$$

Since $\left|\mu_{n}\right| \rightarrow+\infty$ and as $\left(u_{n}\right)$ is bounded (2.2) implies

$$
N_{\Omega}^{\prime}\left(u_{n}\right) \rightarrow 0
$$

because $t_{n} \rightarrow t_{0} \neq 0$. Then, using (2.1), and taking into account that $u_{n} \in \mathcal{N}_{\Omega}$ we have

$$
\begin{aligned}
0 & =\lim _{n \rightarrow+\infty}\left\langle N_{\Omega}^{\prime}\left(u_{n}\right), u_{n}\right\rangle=\lim _{n \rightarrow+\infty} \int_{\Omega}\left[f\left(u_{n}\right) u_{n}-f^{\prime}\left(u_{n}\right) u_{n}^{2}\right] \\
& =\lim _{n \rightarrow+\infty} \int_{\Omega}\left[f\left(u_{n}^{+}\right) u_{n}^{+}-f^{\prime}\left(u_{n}^{+}\right)\left(u_{n}^{+}\right)^{2}\right] .
\end{aligned}
$$

Assume that there exists $\delta>0$ such that

$$
\sup _{y \in \mathbb{R}^{N}} \int_{B_{r}(y)}\left|u_{n}^{+}\right|^{2} \geq \delta
$$

then there exists $\left(y_{n}\right)$ such that

$$
\int_{B_{r}\left(y_{n}\right)}\left|u_{n}^{+}\right|^{2} \geq \delta
$$

arguing as in Lemma 4.2, we observe that two cases may occur, either $\left|y_{n}\right|$ is bounded, or, up to a subsequence, $\left|y_{n}\right| \rightarrow+\infty$. In the first case, there exists $r_{1}>$ 0 such that $\left\|u_{n}^{+}\right\|_{L^{2}\left(B_{r_{1}}(0)\right)}^{2} \geq \delta$. Then, since $u_{n}^{+}$strongly converges to some $v$, in $L_{\text {loc }}^{2}\left(\mathbb{R}^{N}\right)$, it follows that $v \geq 0$, and $v$ satisfies $\|v\|_{L^{2}\left(B_{r_{1}}(0)\right)}^{2} \geq \delta$. Moreover, recalling that $u_{n}^{+} \equiv 0$ outside $\Omega$, we deduce that there exists a measurable set $\Lambda \subseteq \Omega \cap B_{r_{1}}(0)$ with positive measure, such that $v(x)>0$ for $x \in \Lambda$. Then, using (2.5), (2.6) and applying Fatou Lemma, one obtains

$$
\begin{aligned}
0 & =\lim _{n \rightarrow+\infty}-\left\langle N_{\Omega}^{\prime}\left(u_{n}\right), u_{n}\right\rangle=\lim _{n \rightarrow+\infty} \int_{\Omega} f^{\prime}\left(u_{n}^{+}\right)\left(u_{n}^{+}\right)^{2}-f\left(u_{n}^{+}\right) u_{n}^{+} \\
& \geq \lim _{n \rightarrow+\infty} \int_{\Lambda} f^{\prime}\left(u_{n}^{+}\right)\left(u_{n}^{+}\right)^{2}-f\left(u_{n}^{+}\right) u_{n}^{+}=\int_{\Lambda} f^{\prime}(v) v^{2}-f(v) v>0,
\end{aligned}
$$

that is a contradiction, so that $\left|y_{n}\right|$ cannot be bounded, and $\left|y_{n}\right| \rightarrow+\infty$, up to a subsequence. Then, we define $\hat{u}_{n}(\cdot):=u_{n}^{+}\left(\cdot+y_{n}\right)$ and observe that there exists $\hat{u} \geq 0$ almost everywhere in $\mathbb{R}^{N}$, such that $\hat{u}_{n} \rightarrow \hat{u}$ weakly in $H_{0}^{1}(\Omega)$, strongly in $L_{\text {loc }}^{p}\left(\mathbb{R}^{N}\right)$ and almost everywhere. From the strong convergence in $L_{\text {loc }}^{2}\left(\mathbb{R}^{N}\right)$ and using (4.7), one deduces that $\|\hat{u}\|_{L^{2}\left(B_{r_{1}}(0)\right)}^{2} \geq \delta$. Then there exists a measurable set $\Lambda \subseteq B_{r}(0)$ with positive measure, and such that $\hat{u}(x)>0$ for every $x \in \Lambda$. Moreover, as 
$\left|y_{n}\right| \rightarrow \infty$, we can assume that $B_{r}\left(y_{n}\right) \subseteq \Omega$. Then, arguing as in (4.8) we get

$$
\begin{aligned}
0 & =\lim _{n \rightarrow+\infty}-\left\langle N_{\Omega}^{\prime}\left(u_{n}\right), u_{n}\right\rangle \geq \lim _{n \rightarrow+\infty} \int_{B_{r}\left(y_{n}\right)} f^{\prime}\left(u_{n}^{+}\right)\left(u_{n}^{+}\right)^{2}-f\left(u_{n}^{+}\right) u_{n}^{+} \\
& =\lim _{n \rightarrow+\infty} \int_{B_{r}(0)} f^{\prime}\left(\hat{u}_{n}\right) \hat{u}_{n}^{2}-f\left(\hat{u}_{n}\right) \hat{u}_{n} \geq \int_{\Lambda} f^{\prime}(\hat{u}) \hat{u}-f(\hat{u}) \hat{u}>0,
\end{aligned}
$$

so that (2.6) yields again a contradiction, showing that

$$
\lim _{n \rightarrow+\infty} \sup _{y \in \mathbb{R}^{N}} \int_{B_{r}(y)}\left|u_{n}^{+}\right|^{2}=0 .
$$

Then, Lions' Lemma [25] implies

$$
u_{n}^{+} \rightarrow 0 \quad \text { in } L^{p}\left(\mathbb{R}^{N}\right), \text { for any } 2<p<2^{*}
$$

and (2.2) gives

$$
\lim _{n \rightarrow \infty} \int_{\Omega} f\left(u_{n}^{+}\right) u_{n}^{+} \rightarrow 0, \quad \lim _{n \rightarrow \infty} \int_{\Omega} F\left(u_{n}^{+}\right) \rightarrow 0 .
$$

Taking into account that $u_{n} \in \mathcal{N}_{\Omega}$, by (2.1), one deduces that $u_{n}$ strongly converges to zero, which is an evident contradiction with (4.1) implying that $\left(\mu_{n}\right)$ is bounded. As a consequence, there exists $\mu \in \mathbb{R}$ such that, up to a subsequence, $\mu_{n} \rightarrow \mu$. Assume, by contradiction, that $\mu \neq 0$, take $\varphi=u_{n}$ as test function in (4.5) and, recalling that $u_{n} \in \mathcal{N}_{\Omega}$, we have

$$
\left\langle N_{\Omega}^{\prime}\left(u_{n}\right), u_{n}\right\rangle \rightarrow 0 .
$$

From this point we can repeat the same argument, starting from (4.6), to get a contradiction. Then $\mu_{n} \rightarrow 0$ and (4.5) yields the conclusion.

The next lemma studies the asymptotic behavior of a bounded Palais-Smale sequence of $I_{\Omega}$. In the case $f(t)=t^{p}$, the proof is given in [9] (see also Chapter 8 in [39]). However, thanks to hypotheses (2.1), (2.2) the proof can be handled as in the polynomial case arguing as in Chapter 8 in [39]. We will include some details for the sake of clearness.

Lemma 4.5. (Splitting) Assume (2.1), (2.2). Let $\left(u_{n}\right) \in H_{0}^{1}(\Omega)$ be a bounded sequence such that

$$
I_{\Omega}\left(u_{n}\right) \rightarrow d>0 \quad \text { and } \quad I_{\Omega}^{\prime}\left(u_{n}\right) \rightarrow 0 \text { in } H^{-1}(\Omega) .
$$

Replacing $\left(u_{n}\right)$ by a subsequence, if necessary, there exists a solution $u_{0}$ of (1.1), such that the following alternative holds:

either $u_{n} \rightarrow u_{0}$ strongly in $H^{1}\left(\mathbb{R}^{N}\right)$ or there exist a finite sequence $\left(u^{1}, u^{2}, \ldots, u^{k}\right)$ in $H^{1}\left(\mathbb{R}^{N}\right)$ solutions of (2.7) and $k$ sequences of points $\left(y_{n}^{j}\right) \subset \mathbb{R}^{N}, 1 \leq j \leq k$, satisfying:

a) $\left|y_{n}^{j}\right| \rightarrow \infty$ and $\left|y_{n}^{j}-y_{n}^{i}\right| \rightarrow \infty, i \neq j$;

b) $\left\|u_{n}-u_{0}-\sum_{j=1}^{k} u^{j}\left(\cdot-y_{n}^{j}\right)\right\| \rightarrow 0$; 


$$
\text { c) } I_{\Omega}\left(u_{n}\right) \rightarrow I_{\Omega}\left(u_{0}\right)+\sum_{j=1}^{k} I\left(u^{j}\right) \text {. }
$$

Proof. Since $\left(u_{n}\right)$ is bounded, there exists $u_{0} \in H_{0}^{1}(\Omega)$ such that, up to a subsequence, $u_{n} \rightarrow u_{0}$. Then, thanks to the continuity of $f$ and to (2.2), from (4.9) it follows that

$$
I_{\Omega}^{\prime}\left(u_{0}\right)=0 \quad \text { in } H^{-1}(\Omega) .
$$

Let $u_{n}^{1}:=u_{n}-u_{0} \in H_{0}^{1}(\Omega) \subset H^{1}\left(\mathbb{R}^{N}\right)$, and let us show that it results, for $n \rightarrow \infty$,

$$
\begin{aligned}
\left\|u_{n}^{1}\right\|^{2} & =\left\|u_{n}\right\|^{2}-\left\|u_{0}\right\|^{2}+o_{n}(1) ; \\
I\left(u_{n}^{1}\right) & \rightarrow d-I_{\Omega}\left(u_{0}\right) \\
I^{\prime}\left(u_{n}^{1}\right) & \rightarrow 0 \quad \text { in } H^{-1}(\Omega) .
\end{aligned}
$$

The proof of (4.11) is standard. To show (4.12), note that the weak convergence of $\left(u_{n}\right)$ to $u_{0}$ implies $u_{n}^{1} \rightarrow 0$ weakly in $H_{0}^{1}(\Omega)$. Then, applying Theorem 2 in [12] (see also Lemma 8.1 in [39]), it follows that

$$
I\left(u_{n}^{1}\right)-I_{\Omega}\left(u_{n}\right)+I_{\Omega}\left(u_{0}\right)=-\int_{\Omega}\left[F\left(u_{n}^{1}\right)-F\left(u_{n}\right)+F\left(u_{0}\right)\right]+o_{n}(1)=o_{n}(1),
$$

where $o_{n}(1)$ is a quantity tending to zero as $n$ goes to plus infinity. Then, (4.9) yields (4.12). Moreover, (4.13) follows from the following facts: observe that condition (2.2) allows us to use Theorem 2 in [12] or arguing as in Lemma 8.1 in [39], to obtain that $f\left(u_{0}+u_{n}^{1}\right)-f\left(u_{n}^{1}\right) \rightarrow f\left(u_{0}\right)$ in $H^{-1}(\Omega)$. Then, exploiting (4.9), (4.10), it follows, for every $\varphi \in H_{0}^{1}(\Omega)$

$$
\begin{aligned}
\varepsilon_{n}\|\varphi\|_{\Omega} \geq & \left|\left\langle I_{\Omega}^{\prime}\left(u_{n}\right), \varphi\right\rangle\right|=\left|\left\langle I_{\Omega}^{\prime}\left(u_{0}+u_{n}^{1}\right), \varphi\right\rangle\right| \geq\left|\left\langle I_{\Omega}^{\prime}\left(u_{0}\right), \varphi\right\rangle+\left\langle I_{\Omega}^{\prime}\left(u_{n}^{1}\right), \varphi\right\rangle\right| \\
& -\left|\int_{\Omega}\left[f\left(u_{0}+u_{n}^{1}\right)-f\left(u_{0}\right)-f\left(u_{n}^{1}\right)\right] \varphi\right| \\
= & \left|\left\langle I^{\prime}\left(u_{n}^{1}\right), \varphi\right\rangle\right|-\left|\int_{\Omega}\left[f\left(u_{0}+u_{n}^{1}\right)-f\left(u_{0}\right)-f\left(u_{n}^{1}\right)\right] \varphi\right| \\
\geq & \left|\left\langle I^{\prime}\left(u_{n}^{1}\right), \varphi\right\rangle\right|-\varepsilon_{n}\|\varphi\|_{\Omega} .
\end{aligned}
$$

Hence, (4.13) follows. Let us now consider

$$
\delta:=\limsup \sup _{n \rightarrow \infty} \int_{y \in \mathbb{R}^{N}}\left|u_{B_{1}}^{1}\right|^{2} .
$$

If $\delta=0$, it follows from Lions' Lemma [25] that

$$
u_{n}^{1} \rightarrow 0 \quad \text { in } L^{p}\left(\mathbb{R}^{N}\right) \text {, for any } p: 2<p<2^{*} .
$$

On the other hand, as $\left(u_{n}^{1}\right)$ is bounded in $H_{0}^{1}(\Omega)$, we can use (2.2) and (4.14) to deduce that $u_{n}^{1} \rightarrow 0$ strongly in $H_{0}^{1}(\Omega)$ and since $u_{n}^{1} \equiv 0$ outside $\Omega$ it results $u_{n}^{1} \rightarrow 0$ strongly in $H^{1}\left(\mathbb{R}^{N}\right)$. Then the first alternative in the statement of the Lemma holds. 
While, if $\delta>0$, there exists a sequence $\left(y_{n}^{1}\right) \subset \mathbb{R}^{N}$ such that

$$
\int_{B_{1}\left(y_{n}^{1}\right)}\left|u_{n}^{1}\right|^{2}>\frac{\delta}{2} \text {. }
$$

Define a new sequence $\left(v_{n}^{1}\right) \subset H^{1}\left(\mathbb{R}^{N}\right)$ by $v_{n}^{1}:=u_{n}^{1}\left(\cdot+y_{n}^{1}\right)$. Since $\left(u_{n}^{1}\right)$ is bounded in $H^{1}\left(\mathbb{R}^{N}\right)$, then $\left(v_{n}^{1}\right)$ is also bounded and there exists $u^{1} \in H^{1}\left(\mathbb{R}^{N}\right)$, such that $v_{n}^{1} \rightarrow u^{1}$ in $H^{1}\left(\mathbb{R}^{N}\right)$ and $v_{n}^{1} \rightarrow u^{1}$ almost everywhere in $\mathbb{R}^{N}$; then performing a change of variable in (4.15) and applying Fatou Lemma one obtains that

$$
\int_{B_{1}(0)}\left|v_{n}^{1}\right|^{2}>\frac{\delta}{2}, \quad \int_{B_{1}(0)}\left|u^{1}\right|^{2} \geq \frac{\delta}{2}
$$

so that $u^{1} \not \equiv 0$. Moreover, since $u_{n}^{1} \rightarrow 0$ in $H^{1}\left(\mathbb{R}^{N}\right)$, it follows that up to a subsequence we can assume that $\left|y_{n}^{1}\right| \rightarrow \infty$. As shown in [9] (see also Proposition 3.1 in [16] or Chapter 8 in [39]), it results that $I^{\prime}\left(u^{1}\right)=0$ in $H^{-1}\left(\mathbb{R}^{N}\right)$. Indeed, take $\phi \in C_{c}^{\infty}\left(\mathbb{R}^{N}\right)$ and observe that, since $\left|y_{n}^{1}\right| \rightarrow+\infty$ we can find $n_{0}$ such that $\phi_{n}:=\phi\left(x-y_{n}^{1}\right) \in C_{c}^{\infty}(\Omega)$ for every $n \geq n_{0}$; moreover, $\left\|\phi_{n}\right\|_{\Omega} \leq\|\phi\|$. As a consequence, (4.13) yields

$$
\left|\left\langle I^{\prime}\left(u^{1}\right), \phi\right\rangle\right|=\left|\left\langle I^{\prime}\left(v_{n}^{1}\right), \phi\right\rangle\right|+o_{n}(1)=\left|\left\langle I^{\prime}\left(u_{n}^{1}\right), \phi_{n}\right\rangle\right|+o_{n}(1)=o_{n}(1),
$$

so that $u^{1}$ is a solution of (2.7). Defining $u_{n}^{2}(x):=u_{n}^{1}(x)-u^{1}\left(x-y_{n}^{1}\right)$, it results that $u_{n}^{2}\left(\cdot+y_{n}^{1}\right)=v_{n}^{1}-u^{1}$ and repeating for $u_{n}^{2}$ the argument done for $u_{n}^{1}$ we deduce that $\left\|u_{n}^{2}\right\|^{2}=\left\|u_{n}^{1}\right\|^{2}-\left\|u^{1}\right\|^{2}+o_{n}(1), \quad I\left(u_{n}^{2}\right) \rightarrow d-I_{\Omega}\left(u_{0}\right)-I\left(u^{1}\right), \quad I^{\prime}\left(u_{n}^{2}\right) \rightarrow 0$ in $H^{-1}(\Omega)$, so that we go on repeating the argument obtaining $\left(y_{n}^{2}\right)$ satisfying conclusion a) and $u^{2}$, another solution of (2.7). From now on we proceed by iteration. Note that if $u$ is a nontrivial critical point of $I$ and $w$ is the solution of minimum action of (2.7), then

$$
I(u) \geq I(w)>0 .
$$

As a consequence, passing from the step $l$ to the step $l+1$ the action level decreases because in the asymptotic information on the functional $I\left(u_{n}^{l+1}\right)$ it appears $-I\left(u^{l+1}\right)$. Then, taking into account (4.16), the sum must have a finite number of terms, so that, the iteration must be finite and terminate at some index $k \in \mathbb{N}$, yielding also conclusions b) and c).

The following result is a direct consequence of the Splitting Lemma.

Corollary 4.6. Assume (2.1), (2.2), (2.3), (2.4), (2.5), (2.6) and (U). Let $\left(u_{n}\right)$ be a sequence satisfying (4.1) and (4.4) with $d \in(m, 2 m)$. Then $\left(u_{n}\right)$ admits a strongly convergent subsequence.

Proof. Applying Lemma 4.2 we deduce that $\left(u_{n}\right)$ is bounded, moreover Lemma 4.4 allows us to apply Lemma 4.5 to obtain that there exists $u_{0}$ solution of (1.1) such that conclusion $b$ ) holds. Moreover, conclusion $c$ ) and Lemma 4.1 yield

$$
2 m>d=\sum_{j=0}^{k} I\left(u^{j}\right) \geq \begin{cases}k m & \text { if } u_{0} \equiv 0, \\ m_{\Omega}+k m=(k+1) m & \text { if } u_{0} \equiv 0 .\end{cases}
$$


Then, in both cases, $k<2$, i.e. $k=0$ or $k=1$. If $k=1$ and $u_{0} \equiv 0$, it follows that $I_{\Omega}\left(u_{n}\right) \rightarrow I\left(u_{1}\right)=d$ and from hypothesis $(2.1), u_{1}$ is positive, so that, condition $(U)$ yields $u_{1}=w$ and $d=I\left(u_{1}\right)=m$, which contradicts the hypothesis. Also note that the hypothesis $d<2 m$ implies that it is not possible that $k=1$ and $u_{0} \not \equiv 0$. Then, the only possibility is that $k=0$, that is $d=I\left(u_{0}\right)=I_{\Omega}\left(u_{0}\right)$ and Lemma 4.5 implies $u_{n}$ strongly converges to $u_{0}$.

\section{The Linking Argument and Proof of the Main Results}

We will prove our existence results by applying the Linking theorem on the manifold $\mathcal{N}_{\Omega}$ (see Theorem 8.22 in [5] or Theorem 8.4 in [37] joint with Lemma 5.14 and 5.15 in [39]). This argument has already been used in [4] to prove existence results for linearly coupled semi-linear non-autonomous equations in $\mathbb{R}^{N}$. In order to define the linking sets, we will make use of the properties of a barycenter function, already adopted in [9] and then often used when building solution at higher action level than the least one (see for example [4, 18] or [8] for an interesting generalization). Here, we will follow the notation in [18] (see also [4]). For every $u \in H^{1}\left(\mathbb{R}^{N}\right) \backslash\{0\}$, the following maps are well defined

$$
\begin{gathered}
\mu(u)(x):=\frac{1}{\left|B_{1}(x)\right|} \int_{B_{1}(x)}|u(y)| d y, \quad \mu(u) \in L^{\infty} \cap C^{0}(0,+\infty), \\
\hat{u}(x):=\left[\mu(u)(x)-\frac{\|\mu(u)\|_{\infty}}{2}\right]^{+}, \quad \hat{u} \in C_{0}\left(\mathbb{R}^{N}\right) .
\end{gathered}
$$

Then, the barycenter of a function $u \in H^{1}\left(\mathbb{R}^{N}\right) \backslash\{0\}$ defined by

$$
\beta(u)=\frac{1}{\|\hat{u}\|_{1}} \int_{\mathbb{R}^{N}} x \hat{u}(x) d x
$$

is a continuous function enjoying the following properties

$$
\begin{gathered}
\beta(u(\cdot-y))=\beta(u)+y \quad \forall y \in \mathbb{R}^{N}, \\
\beta(T u)=\beta(u) \quad \forall T>0 .
\end{gathered}
$$

One of the linking set is the following subset of $\mathcal{N}_{\Omega}$

$$
S:=\left\{u \in H_{0}^{1}(\Omega),: u \in \mathcal{N}_{\Omega}, \beta(u)=0\right\} .
$$

Remark 5.1. Notice that $S \neq \emptyset$. Indeed, first note that, from the properties of the function $w$ we can find $r_{0}>0$ such that $\left\{x \in \mathbb{R}^{N}: \mu(w)>\|\mu(w)\|_{\infty} / 2\right\}=B_{r_{0}}(0)$. Then, take $\theta \in \mathbb{R}^{N}$ with $|\theta|=1, R>4 \rho r_{0}$ and the functions $\eta_{1}$ and $\eta_{2}$ defined as $\eta_{1}(x)=1-\xi(4 \rho(x-R \theta) / R)$ and $\eta_{2}(x)=1-\xi(4 \rho(x+R \theta) / R)$. Choose the function $z_{R}=w^{R \theta} \eta_{1}+w^{-R \theta} \eta_{2}$. The properties of the function $\eta_{1}$ imply that $\eta_{1} w^{R \theta}<w^{R \theta}$ so that $\mu\left(\eta_{1} w^{R \theta}\right)<\mu\left(w^{R \theta}\right)$ and it results

$$
\sup _{\mathbb{R}^{N} \backslash B_{R / 4 \rho}(R \theta)} \mu\left(\eta_{1} w^{R \theta}\right)<\sup _{B_{R / 4 \rho}(R \theta)} \mu\left(\eta_{1} w^{R \theta}\right)=\sup _{B_{R / 4 \rho}(R \theta)} \mu\left(w^{R \theta}\right)=\mu\left(w^{R \theta}\right)(R \theta),
$$


where we have taken into account that $\mu(0)>\mu(x)$ for every $x \in \mathbb{R}^{N} \backslash\{0\}$. As a consequence of the previous facts,

$$
\left\{x \in \mathbb{R}^{N}: \mu\left(\eta_{1} w^{R \theta}\right)>\frac{\left\|\mu\left(\eta_{1} w^{R \theta}\right)\right\|_{\infty}}{2}\right\} \subset B_{R / 4 \rho}(R \theta) .
$$

Arguing analogously for $\eta_{2} w^{-R \theta}$, one obtains ${\widehat{\eta_{1} w}}^{R \theta}=\widehat{w}^{R \theta}$ and ${\widehat{\eta_{2} w}}^{-R \theta}=\widehat{w}^{-R \theta}$. Moreover, observe that $\mathcal{G}_{\Omega}\left(z_{R}\right)=\mathcal{G}_{\Omega}\left(w^{R \theta}\right)+\mathcal{G}_{\Omega}\left(w^{-R \theta}\right)+o(R)=2 \mathcal{G}_{\Omega}(w)+o(R)$, where $o(R)$ is a quantity tending to zero as $R$ tends to infinity. Then $z_{R} \in A_{\Omega}$ for $R$ sufficiently large. Therefore, $v=T_{\Omega}\left(z_{R}\right) z_{R} \in \mathcal{N}_{\Omega}$ and from (5.2) it results $\beta(v)=\beta\left(z_{R}\right)$. In addition taking into account that $B_{R / 2}(R \theta) \cap B_{R / 2}(-R \theta)=\emptyset$, we obtain $\hat{z}_{R}={\widehat{\eta_{1} w}}^{R \theta}+{\widehat{\eta_{2} w}}^{-R \theta}$. Then it results

$$
\beta(v)=\beta\left(z_{R}\right)=\beta\left(\eta_{1} w^{R \theta}\right)+\beta\left(\eta_{2} w^{-R \theta}\right)=\beta\left(w^{R \theta}\right)+\beta\left(w^{-R \theta}\right)=0,
$$

showing that $z_{R} \in S$.

Lemma 5.2. Assume (2.1), (2.2), (2.3), (2.4), (2.5), (2.6). Let $b=\inf _{S} I_{\Omega}(u)$, then $b>m_{\Omega}$.

Proof. It is clear that $b \geq m_{\Omega}$. To prove the strict inequality we shall argue by contradiction. Suppose $b=m_{\Omega}$, then there exists a sequence $\left(v_{n}\right) \subset \mathcal{N}_{\Omega}$ such that $\beta\left(v_{n}\right)=0,\left\langle I_{\Omega}^{\prime}\left(v_{n}\right), v_{n}\right\rangle=0$ and $I_{\Omega}\left(v_{n}\right) \rightarrow m_{\Omega}$; moreover, $\left(v_{n}\right)$ is not relatively compact because $m_{\Omega}$ is not attained. By Ekeland Variational Principle (Theorem 8.5 in [39]) applied to the closed manifold $\mathcal{N}_{\Omega}$, there exists another sequence $\left(\tilde{v}_{n}\right) \subset$ $\mathcal{N}_{\Omega}$ such that:

$$
I_{\Omega}\left(\tilde{v}_{n}\right) \rightarrow m_{\Omega}, \quad \nabla_{\mathcal{N}_{\Omega}} I_{\Omega}\left(\tilde{v}_{n}\right) \rightarrow 0, \quad\left\|\tilde{v}_{n}-v_{n}\right\|_{\Omega} \rightarrow 0 .
$$

From Lemma $4.2\left(v_{n}\right)$ and $\left(\tilde{v}_{n}\right)$ are bounded. Moreover, Lemma 4.4 implies that

$$
I_{\Omega}^{\prime}\left(\tilde{v}_{n}\right) \rightarrow 0 .
$$

By exploiting hypothesis (2.2) we deduce that that $I_{\Omega}^{\prime \prime}$ maps bounded sets of $H_{0}^{1}(\Omega)$ in bounded sets, then the mean value Theorem implies that the following inequality holds for every $\phi \in H_{0}^{1}(\Omega)$,

$$
\left|\left\langle I_{\Omega}^{\prime}\left(\tilde{v}_{n}\right)-I_{\Omega}^{\prime}\left(v_{n}\right), \phi\right\rangle\right| \leq K\left\|\tilde{v}_{n}-v_{n}\right\|_{\Omega}\|\phi\|_{\Omega} .
$$

Taking the supremum on $\phi$ and using (5.5), it follows that also $\left(v_{n}\right)$ is a Palais-Smale sequence. Therefore, from Lemma 4.5 and since $m_{\Omega}$ is not attained, we deduce that conclusions $a$ )-c) hold. In particular, conclusion $c$ ), (5.4) and Lemma 4.1 imply

$$
m=I_{\Omega}\left(u_{0}\right)+\sum_{j=1}^{k} I\left(u^{j}\right) \geq I_{\Omega}\left(u_{0}\right)+k m \geq k m,
$$

where the last inequality is implied by the fact that, either $u_{0} \equiv 0$ so that $I_{\Omega}\left(u_{0}\right)=0$, or $u_{0} \in \mathcal{N}_{\Omega}$ so that $I\left(u_{0}\right)>m_{\Omega}>0$. Then, $k$ has to be equal to one, $u_{0}$ has to be trivial and $u^{1}=w$. This and conclusion $\left.b\right)$ of Lemma 4.5 yield $v_{n}(\cdot)-w(\cdot-$ $\left.y_{n}\right) \rightarrow 0$, strongly in $H^{1}\left(\mathbb{R}^{N}\right)$, where $y_{n} \in \mathbb{R}^{N},\left|y_{n}\right| \rightarrow \infty$. Calculating the barycenter 
function of $v_{n}(x)$ and $w\left(x-y_{n}\right)$, we have, as $\beta$ is a continuous function, $w$ is radially symmetric, and using (5.1)

$$
0=\beta\left(v_{n}\right)=\beta\left(w\left(\cdot-y_{n}\right)\right)+o(1)=\beta(w)+y_{n}+o(1)=y_{n}+o(1)
$$

where $o(1)$ a quantity tending to zero as $n$ goes to plus infinity. This immediately gives a contradiction as $\left|y_{n}\right| \rightarrow+\infty$.

In order to define the other linking set, we argue as in [18] (see also Section 7 in [4]) and we take $x_{0}$ and $y$ as in (3.15) and $R>\max \left\{R_{0}, R_{1}\right\}$ where $R_{0}$ and $R_{1}$ are introduced in Lemmas 3.5 and 3.8. Let us define the function

$$
z: \partial B_{2}\left(x_{0}\right) \times[0,1] \mapsto \overline{B_{2}\left(x_{0}\right)}, \quad \text { by } \quad z(y, t):=t y+(1-t) x_{0} .
$$

Notice that $z$ is a homeomorphism from $\partial B_{2}\left(x_{0}\right) \times(0,1]$ to $\overline{B_{2}\left(x_{0}\right)} \backslash\left\{x_{0}\right\}$, so that for every point $\tilde{z} \in \overline{B_{2}\left(x_{0}\right)}$ there is a unique pair $(y, t) \in \partial B_{2}\left(x_{0}\right) \times(0,1]$, such that $\tilde{z}=z(y, t) \neq x_{0}$. Therefore, we can define the operator $\Psi_{R}: \overline{B_{2}\left(x_{0}\right)} \mapsto \mathcal{N}_{\Omega}$ by the values that it takes on $\partial B_{2}\left(x_{0}\right) \times[0,1]$ as follows

$$
\Psi_{R}[z]= \begin{cases}\Psi_{R}[z(y, t)]=\Psi_{R}[y, t]=\Pi_{\mathcal{N}_{\Omega}}\left(U_{t}^{R}\right) & \text { if } z \neq x_{0}, \\ \Psi_{R}[y, 0]=\Pi_{\mathcal{N}_{\Omega}}\left(\Phi^{R x_{0}}\right) & \text { if } z=x_{0},\end{cases}
$$

where $U_{t}^{R}$ is given in (3.15) and $\Pi_{\mathcal{N}_{\Omega}}$ is defined in (3.6). Notice that $\Psi_{R}$ is well defined thanks to Lemma 3.8. In order to apply the Linking Theorem on $\mathcal{N}_{\Omega}$ we recall that $S$ is defined in (5.3) and we set

$$
\begin{gathered}
Q:=\Psi_{R}\left(\overline{B_{2}\left(x_{0}\right)}\right) \\
\mathcal{H}:=\left\{h \in C^{0}\left(Q, \mathcal{N}_{\Omega}\right),\left.h\right|_{\partial Q}=\mathrm{id}\right\}, \quad d:=\inf _{h \in \mathcal{H}} \sup _{u \in Q} I_{\Omega}(h(u)) .
\end{gathered}
$$

The linking geometrical structure is showed in the next lemma.

Lemma 5.3. Assume conditions (2.1), (2.2), (2.3), (2.4), (2.5), (2.6). Let $Q$ and $\mathcal{H}$ be defined in (5.7) (5.8). Then, for $R$ sufficiently large, it results

$$
\begin{aligned}
\partial Q \cap S & =\emptyset, \\
h(Q) & \cap S \neq \emptyset \quad \forall h \in \mathcal{H}, \\
\sup _{\partial Q} I_{\Omega}(u) & <\inf _{S} I_{\Omega}(u) .
\end{aligned}
$$

Proof. First of all, notice that $\Psi_{R}: \overline{B_{2}\left(x_{0}\right)} \mapsto \Psi_{R}\left(\overline{B_{2}\left(x_{0}\right)}\right)$ is a continuous bijection defined on a compact set. Indeed, let us first show that $\Psi_{R}$ is injective in $\overline{B_{2}\left(x_{0}\right)} \backslash$ $\left\{x_{0}\right\}$. In order to do this, let us consider $z_{1}, z_{2} \in \overline{B_{2}\left(x_{0}\right)} \backslash\left\{x_{0}\right\}$ such that $\Psi_{R}\left(z_{1}\right)=$ $\Psi_{R}\left(z_{2}\right)$. Since $z(y, t)$ is injective, this amounts to consider $\left(y_{1}, t_{1}\right),\left(y_{2}, t_{2}\right) \in \partial B_{2}\left(x_{0}\right) \times$ $(0,1]$ such that $\Psi_{R}\left[z\left(y_{1}, t_{1}\right)\right]=\Psi_{R}\left[z\left(y_{2}, t_{2}\right)\right]$. Taking into account Remark (3.6), and using the notation

$$
T_{i}(R)=T_{\Omega}\left(t_{i} \Phi^{R y_{i}}+\left(1-t_{i}\right) \Phi^{R x_{0}}\right),
$$

this is equivalent to have

$$
T_{1}(R)\left(t_{1} \Phi^{R y_{1}}+\left(1-t_{1}\right) \Phi^{R x_{0}}\right)=T_{2}(R)\left(t_{2} \Phi^{R y_{2}}+\left(1-t_{2}\right) \Phi^{R x_{0}}\right) .
$$


Suppose that $\left|y_{1}-y_{2}\right|=a>0$. Since $\left|y_{i}-x_{0}\right|=2$ for $i=1,2$, (3.3) implies that $w\left(R\left(y_{2}-y_{1}\right)\right)=o(R)$ and $w\left(R\left(y_{i}-x_{0}\right)\right)=o(R)$, where $o(R)$ is a quantity tending to zero as $R$ tends to infinity. Choosing $x=R y_{1}$ with $R>2 \rho$, and recalling (3.8), one obtains $T_{1}(R)\left(t_{1} w(0)+\left(1-t_{1}\right) o(R)\right)=T_{2}(R) o(R)$. Then, (3.16) implies that $T_{1}(R) t_{1} w(0)=o(R)$. Since $w(0)>0$ and $t_{1} \in(0,1]$, this implies that $T_{1}(R)=o(R)$. Arguing as in the proof of Lemma 3.8, one can reach a contradiction, so that $\mid y_{1}-$ $y_{2} \mid=0$, i.e. $y_{1}=y_{2}$. In order to prove that $t_{1}=t_{2}$ let us choose $x=R y=R y_{1}=R y_{2}$, take into account (3.2) and that $R>2 \rho$ to obtain

$$
T_{1}(R)\left[t_{1} w(0)+\left(1-t_{1}\right) w(2 R)\right]=T_{2}(R)\left(t_{2} w(0)+\left(1-t_{2}\right) w(2 R)\right) .
$$

On the other hand, choosing $x=R x_{0}$ we get

$$
T_{1}(R)\left[t_{1} w(2 R)+\left(1-t_{1}\right) w(0)\right]=T_{2}(R)\left[t_{2} w(2 R)+\left(1-t_{2}\right) w(0)\right] .
$$

Then

$$
\frac{t_{1} w(0)+\left(1-t_{1}\right) w(2 R)}{t_{1} w(2 R)+\left(1-t_{1}\right) w(0)}=\frac{t_{2} w(0)+\left(1-t_{2}\right) w(2 R)}{t_{2} w(2 R)+\left(1-t_{2}\right) w(0)}
$$

Since the function $h(t)=a t+b(1-t) /[b t+a(1-t)]$ is injective, the above equality implies that $t_{1}=t_{2}$. Then, we have shown that $\Psi$ is injective in $\overline{B_{2}\left(x_{0}\right)} \backslash\left\{x_{0}\right\}$. Now, take $z \in \overline{B_{2}\left(x_{0}\right)}$ such that $\Psi_{R}(z)=\Psi_{R}\left(x_{0}\right)$, that is

$$
T_{\Omega}\left(t \Phi^{R y}+(1-t) \Phi^{R x_{0}}\right)\left[t \Phi^{R y}+(1-t) \Phi^{R x_{0}}\right]=T_{\Omega}\left(\Phi^{R x_{0}}\right) \Phi^{R x_{0}} .
$$

Choosing $x=R y$ and arguing as before, one obtains that $z=x_{0}$ proving that $\Psi_{R}$ is injective in $\overline{B_{2}\left(x_{0}\right)}$. As a consequence, $\Psi_{R}$ is an homeomorphism and $\partial Q=$ $\Psi_{R}\left(\partial B_{2}\left(x_{0}\right)\right)$.

In order to show that (5.9) holds, we observe that, since $w$ is radially symmetric, positive and decreasing in $(0,+\infty)$, also $\mu(w)$ is decreasing with respect to $|x|$; Moreover, as proved in Theorem 2.1 in [8] $\mu(w) \rightarrow 0$ as $|x| \rightarrow+\infty$, then, arguing as in Remark 5.1, we obtain that there exists a unique $r_{0}>0$ such that for every $|x|=r_{0}, \mu(w)(x)=\|\mu(w)\|_{\infty} / 2$ and by (5.1) the set

$$
E(w):=\left\{x \in \mathbb{R}^{N}, \mu(w)(x) \geq \frac{\|\mu(w)\|_{\infty}}{2}\right\}
$$

is such that

$$
E(w)=B_{r_{0}}(0) \Rightarrow E(w(\cdot-R y))=B_{r_{0}}(R y),
$$

for every $R \in \mathbb{R}^{+}$. Let us fix $R$ such that $R>2 \rho+1+r_{0}$ and, as $y \in \partial B_{2}\left(x_{0}\right)$, it results that $|x|>2 \rho+r_{0}$ for every $x \in B_{1}(R y)$. Then, exploiting (3.2) we obtain

$$
\begin{aligned}
\mu\left(\Phi^{R y}\right)(R y) & =\frac{1}{\left|B_{1}(R y)\right|} \int_{B_{1}(R y)} \xi(x) w(x-R y) d x=\frac{1}{\left|B_{1}(R y)\right|} \int_{B_{1}(R y)} w(x-R y) d x \\
& =\frac{1}{\left|B_{1}(0)\right|} \int_{B_{1}(0)} w(\sigma) d \sigma=\mu(w)(0)=\|\mu(w)\|_{\infty} .
\end{aligned}
$$

Since, $\|\xi\|_{\infty} \leq 1$, it results

$$
\left|\mu\left(\Phi^{R y}\right)(x)\right| \leq|\mu(w)(x-R y)| \leq\|\mu(w)\|_{\infty},
$$


showing that

$$
\left\|\mu\left(\Phi^{R y}\right)\right\|_{\infty}=\left|\mu\left(\Phi^{R y}\right)(R y)\right|=\|\mu(w)\|_{\infty} .
$$

In addition, for every $x \in B_{r_{0}}(R y)$, any $z \in B_{1}(x)$ satisfies $|z|>2 \rho$, showing that $B_{1}(x) \subseteq \mathbb{R}^{N} \backslash B_{2 \rho}(0)$, and using again (3.2), we have

$$
\begin{aligned}
\mu\left(\Phi^{R y}\right)(x) & =\frac{1}{\left|B_{1}(x)\right|} \int_{B_{1}(x)} \xi(z) w(z-R y) d z=\frac{1}{\left|B_{1}(x)\right|} \int_{B_{1}(x)} w(z-R y) d z \\
& =\mu(w)(x-R y) .
\end{aligned}
$$

Recalling (5.12) we have that, for every $x \in B_{r_{0}}(R y), \mu(w)(x-R y)>\|\mu(w)\|_{\infty} / 2$, yielding

$$
\mu\left(\Phi^{R y}\right)(x)>\frac{1}{2}\|\mu(w)\|_{\infty} \quad \text { for every } x \in B_{r_{0}}(R y),
$$

so that $\widehat{\Phi}^{R y} \neq 0$ if $x \in B_{r_{0}}(R y)$. If $x \notin B_{r_{0}}(R y)$ recalling (5.12), (5.13) and (5.14), it results

$$
\mu\left(\Phi^{R y}\right) \leq \mu(w(\cdot-R y))<\frac{1}{2}\|\mu(w(\cdot-R y))\|_{\infty}=\frac{1}{2}\left\|\mu\left(\Phi^{R y}\right)\right\|_{\infty} .
$$

Therefore, $\widehat{\Phi}^{R y} \neq 0$ if and only if $x \in B_{r_{0}}(R y)$, but, in this set $\xi \equiv 1$, so that $\widehat{\Phi}^{R y}=\widehat{w}(\cdot-R y)$ and hence

$$
\beta\left(\Phi^{R y}\right)=\beta(w(\cdot-R y))=R y,
$$

showing (5.9). In order to prove (5.10), for every $h \in \mathcal{H}$, let us take $V: \overline{B_{2}\left(x_{0}\right)} \mapsto$ $\mathbb{R}^{N}$ given by $V(z)=\left(\beta \circ h \circ \Psi_{R}\right)(z)$. If $z \in \partial B_{2}\left(x_{0}\right), z=z(y, 1)$, then, it results $\Psi_{R}(z) \in \partial Q$, so that $h\left(\Psi_{R}(z)\right)=\Psi_{R}[y, 1]$, then (5.2) and (5.15) yield

$$
V(z)=\beta\left(\Psi_{R}[y, 1]\right)=\beta\left(\Psi_{R}\left(\Phi^{R y}\right)\right)=\beta\left(T_{\Omega}\left(\Phi^{R y}\right) \Phi^{R y}\right)=\beta\left(\Phi^{R y}\right)=R y .
$$

Then, by Brouwer Theorem, there exists $z_{0} \in B_{2}\left(x_{0}\right)$ such that $V\left(z_{0}\right)=0$, i.e. $h\left(\Psi_{R}\left(z_{0}\right)\right) \in S$, and, as by definition $\Psi_{R}\left(z_{0}\right) \in Q$, (5.10) follows.

In order to show (5.11), let us first observe that $\partial Q=\Psi_{R}\left(\partial B_{2}\left(x_{0}\right)\right)=\Psi_{R}[y, 1]$; moreover,

$$
I_{\Omega}\left(\Psi_{R}[y, 1]\right)=I_{\Omega}\left(\Pi_{\mathcal{N}_{\Omega}}\left(\Phi^{R y}\right)\right)=I_{\Omega}\left(T_{\Omega}\left(\Phi^{R y}\right) \Phi^{R y}\right) .
$$

From (3.10) and (3.12) it follows that $I_{\Omega}\left(\Psi_{R}[y, 1]\right) \rightarrow m$ as $R \rightarrow+\infty$; then, Lemma 4.1 and Lemma 5.2 imply that, fixing $R$ sufficiently large, (5.11) holds.

Lemma 5.4. Assume (2.1), (2.2), (2.3), (2.4). There exists $R_{2}>\max \left\{R_{0}, R_{1}\right\}$ such that

$$
\max _{Q} I_{\Omega}(u)<2 m_{\Omega}, \quad \text { for every } R \geq R_{2}
$$

Proof. In the proof we will use the notation, introduced in Lemma 3.9

$$
T_{t}^{R}=T_{\Omega}\left(U_{t}^{R}\right)
$$

where $T(u)$ is defined in Lemma 3.5. Recalling (5.7), (5.6), and taking into account (3.15), it is sufficient to show that there exists $R_{2}$ sufficiently large, such that

$$
I_{\Omega}\left(\Pi_{\mathcal{N}_{\Omega}}\left(U_{t}^{R}\right)\right)=I_{\Omega}\left(T_{t}^{R} U_{t}^{R}\right)<2 m_{\Omega}, \quad \forall R \geq R_{2}, \forall(y, t) \in \partial B_{2}\left(x_{0}\right) \times[0,1] .
$$


It results

$$
\begin{aligned}
I_{\Omega}\left(T_{t}^{R} U_{t}^{R}\right)= & \frac{\left(T_{t}^{R} t\right)^{2}}{2}\left\|\Phi^{R y}\right\|_{\Omega}^{2}+\frac{\left(T_{t}^{R}(1-t)\right)^{2}}{2}\left\|\Phi^{R x_{0}}\right\|_{\Omega}^{2}+\left(T_{t}^{R}\right)^{2} t(1-t) \int_{\Omega} \nabla \Phi^{R y} \nabla \Phi^{R x_{0}} \\
& +\left(T_{t}^{R}\right)^{2} t(1-t) \lambda \int_{\Omega} \Phi^{R y} \Phi^{R x_{0}}-\int_{\Omega} F\left(T_{t}^{R} t \Phi^{R y}+T_{t}^{R}(1-t) \Phi^{R x_{0}}\right) .
\end{aligned}
$$

By adding and subtracting $I\left(T_{t}^{R} t w^{R y}\right)+I\left(T_{t}^{R}(1-t) w^{R x_{0}}\right)$ and taking into account (3.9) and Lemma 3.8 one obtains

$$
\begin{aligned}
I_{\Omega}\left(T_{t}^{R} U_{t}^{R}\right)= & I\left(T_{t}^{R} t w^{R y}\right)+I\left(T_{t}^{R}(1-t) w^{R x_{0}}\right)+\int_{\mathbb{R}^{N}}\left[F\left(T_{t}^{R} t w^{R y}\right)-F\left(T_{t}^{R} t \Phi^{R y}\right)\right] \\
& -\int_{\mathbb{R}^{N}}\left[F\left(T_{t}^{R} U_{t}^{R}\right)-F\left(T_{t}^{R} t \Phi^{R y}\right)-F\left(T_{t}^{R}(1-t) \Phi^{R x_{0}}\right)\right] \\
& +\left(T_{t}^{R}\right)^{2} t(1-t) \int_{\Omega} \nabla \Phi^{R y} \nabla \Phi^{R x_{0}} \lambda \Phi^{R y} \Phi^{R x_{0}}+o\left(\varepsilon_{R}\right) \\
& +\int_{\mathbb{R}^{N}}\left[F\left(T_{t}^{R}(1-t) w^{R x_{0}}\right)-F\left(T_{t}^{R}(1-t) \Phi^{R x_{0}}\right)\right]
\end{aligned}
$$

Moreover, using (3.11) and (3.16), one obtains

$$
\begin{aligned}
I_{\Omega}\left(T_{t}^{R} U_{t}^{R}\right) & \leq I\left(T_{t}^{R} t w^{R y}\right)+I\left(T_{t}^{R}(1-t) w^{R x_{0}}\right)+o\left(\varepsilon_{R}\right) \\
& +\left(T_{t}^{R}\right)^{2} t(1-t) \int_{\Omega} \nabla \Phi^{R y} \nabla \Phi^{R x_{0}}+\lambda \Phi^{R y} \Phi^{R x_{0}} \\
& -\int_{\mathbb{R}^{N}}\left[F\left(T_{t}^{R} U_{t}^{R}\right)-F\left(T_{t}^{R} t \Phi^{R y}\right)-F\left(T_{t}^{R}(1-t) \Phi^{R x_{0}}\right)\right] .
\end{aligned}
$$

Applying Lemmas 3.7 and 3.9 we deduce

$$
\begin{gathered}
I_{\Omega}\left(T_{t}^{R} U_{t}^{R}\right) \leq I\left(T_{t}^{R} t w^{R y}\right)+I\left(T_{t}^{R}(1-t) w^{R x_{0}}\right)+o\left(\varepsilon_{R}\right) \\
+\left(T_{t}^{R}\right)^{2} t(1-t)\left[\frac{1}{2} \int_{\mathbb{R}^{N}} f\left(w^{R y}\right) w^{R x_{0}}+\frac{1}{2} \int_{\mathbb{R}^{N}} f\left(w^{R x_{0}}\right) w^{R y}\right] \\
-\int_{\mathbb{R}^{N}}\left[f\left(T_{t}^{R} t \Phi^{R y}\right) T_{t}^{R}(1-t) \Phi^{R x_{0}}+f\left(T_{t}^{R}(1-t) \Phi^{R x_{0}}\right) T_{t}^{R} t \Phi^{R y}\right] .
\end{gathered}
$$

Applying Lemmas 3.8 and 3.11 (see (3.33)) in the last term one gets

$$
\begin{aligned}
I_{\Omega}\left(T_{t}^{R} U_{t}^{R}\right) \leq & I\left(T_{t}^{R} t w^{R y}\right)+I\left(T_{t}^{R}(1-t) w^{R x_{0}}\right)+o\left(\varepsilon_{R}\right) \\
& +\left(T_{t}^{R}\right)^{2} t(1-t)\left[\frac{1}{2} \int_{\mathbb{R}^{N}} f\left(w^{R y}\right) w^{R x_{0}}+\frac{1}{2} \int_{\mathbb{R}^{N}} f\left(w^{R x_{0}}\right) w^{R y}\right] \\
& -\int_{\mathbb{R}^{N}}\left[f\left(T_{t}^{R} t w^{R y}\right) T_{t}^{R}(1-t) w^{R x_{0}}+f\left(T_{t}^{R}(1-t) w^{R x_{0}}\right) T_{t}^{R} t w^{R y}\right] .
\end{aligned}
$$


We use (3.34) with the choices $\tau=T_{t}^{R} t, \tau=T_{t}^{R}(1-t)$ and use (3.16) to get that there exists a positive constant $C_{1}$ such that

$$
\begin{aligned}
I_{\Omega}\left(T_{t}^{R} U_{t}^{R}\right) \leq & I\left(T_{t}^{R} t w^{R y}\right)+I\left(T_{t}^{R}(1-t) w^{R x_{0}}\right)+o\left(\varepsilon_{R}\right) \\
- & \frac{1}{2} \int_{\mathbb{R}^{N}}\left[f\left(T_{t}^{R} t w^{R y}\right) T_{t}^{R}(1-t) w^{R x_{0}}+f\left(T_{t}^{R}(1-t) w^{R x_{0}}\right) T_{t}^{R} t w^{R y}\right] \\
& +C_{1}\left[\left|t T_{t}^{R}-1\right|+\left|(1-t) T_{t}^{R}-1\right|\right] \varepsilon_{R} .
\end{aligned}
$$

Choosing $\left(\tau_{1}, \tau_{2}\right)=\left(T_{t}^{R}(1-t), T_{t}^{R} t\right)$ in the first inequality in Lemma 3.10, and $\left(\tau_{1}, \tau_{2}\right)=\left(T_{t}^{R} t, T_{t}^{R}(1-t)\right)$ in the second one, we obtain (thanks to (3.16))

$$
\begin{aligned}
& I_{\Omega}\left(T_{t}^{R} U_{t}^{R}\right) \leq I\left(T_{t}^{R} t w^{R y}\right)+I\left(T_{t}^{R}(1-t) w^{R x_{0}}\right)+o\left(\varepsilon_{R}\right) \\
& \quad-\varepsilon_{R} C_{1}\left[\left|t T_{t}^{R}-1\right|+\left|(1-t) T_{t}^{R}-1\right|\right]-\varepsilon_{R} C_{2} \min \left\{t T_{t}^{R},(1-t) T_{t}^{R}\right\},
\end{aligned}
$$

with $C_{2}$ a positive constant. In view of (3.17), there exists $\delta>0$ such that for every $t \in\left(\frac{1}{2}-\delta, \frac{1}{2}+\delta\right)$, it results

$$
C_{1}\left[\left|t T_{t}^{R}-1\right|+\left|(1-t) T_{t}^{R}-1\right|\right]+C_{2} \min \left\{t T_{t}^{R},(1-t) T_{t}^{R}\right\} \geq \frac{C_{2}}{2} .
$$

Moreover, the real valued function $h_{v}$ defined as

$$
h_{v}(p):=\frac{p^{2}}{2} f(v) v-F(p v), \quad I(p v)=\int_{\mathbb{R}^{N}} h_{v}(p) .
$$

achieves the unique maximum value for $p=1$ (see for more details Lemma 4.2 or Proposition 3.20 in [28]) then, as $w \in \mathcal{N}$, it results

$$
I\left(T_{t}^{R} t w^{R y}\right) \leq \int_{\mathbb{R}^{N}} \frac{1}{2} f(w) w-F(w)=I(w)=m .
$$

The same conclusion holds for the term $\left.I\left(T_{t}^{R}(1-t) w^{R x_{0}}\right)\right)$, so that from (5.18) it follows

$$
I_{\Omega}\left(T_{t}^{R} U_{t}^{R}\right) \leq 2 m-\frac{C_{2}}{2} \varepsilon_{R}+o\left(\varepsilon_{R}\right)
$$

which, recalling Lemma 4.1, shows the conclusion if $t \in\left(\frac{1}{2}-\delta, \frac{1}{2}+\delta\right)$.

In the other case, namely when $|t-1 / 2|>\delta$, we can fix a positive constant $\sigma_{0}$ such that

$$
\left|t T_{t}^{R}-1\right|>\sigma_{0} \quad \text { or } \quad\left|(1-t) T_{t}^{R}-1\right|>\sigma_{0} .
$$

Indeed, if (5.21) were not true, we could find positive sequences $\left(\sigma_{n}\right),\left(t_{n}\right),\left(T_{n}\right)$ such that, up to subsequences, $\sigma_{n} \rightarrow 0, t_{n} \rightarrow t_{0} \in[0,1], T_{n} \rightarrow T_{0} \in[0, L]$ (thanks to $(3.16))$, and

$$
\left|t_{n} T_{n}-1\right| \leq \sigma_{n} \quad \text { and } \quad\left|\left(1-t_{n}\right) T_{n}-1\right| \leq \sigma_{n} .
$$

Since $\sigma_{n} \rightarrow 0$, it results $\left(1-t_{0}\right) T_{0}=1$ and $t_{0} T_{0}=1$, so that $T_{0} \neq 0$ and $t_{0} T_{0}=$ $\left(1-t_{0}\right) T_{0}$, which implies that $t_{0}=1 / 2$, that is not possible since $\left|t_{0}-1 / 2\right|>\delta$. 
Then (5.21) is true and we also claim that there exists a positive constant $\alpha_{0}$ such that

$$
\int_{\mathbb{R}^{N}} h_{w}(p)<m-\alpha_{0} \forall p \in[0, L]:|p-1|>\sigma_{0},
$$

where $h_{v}(p)$ is defined in (5.19), Indeed, by contradiction, there exists a sequence $\alpha_{n}$ converging to zero, and a sequence of point $p_{n} \in[0, L]$ such that

$$
\left|p_{n}-1\right|>\sigma_{0}, \quad \text { and } \quad \int_{\mathbb{R}^{N}} h_{w}\left(p_{n}\right) \geq m-\alpha_{n} .
$$

Up to a subsequence, there exists $p_{0} \in[0, L]$ such that $p_{n} \rightarrow p_{0}$. Taking limit in (5.23) we find

$$
\left|p_{0}-1\right|>\sigma_{0}, \quad \text { and } \quad \int_{\mathbb{R}^{N}} h_{w}\left(p_{0}\right) \geq m .
$$

Since $\bar{p}=1$ is the unique maximum point of $h_{w}(p)$ there results

$$
m=\int_{\mathbb{R}^{N}} h_{w}(1) \geq \int_{\mathbb{R}^{N}} h_{w}\left(p_{0}\right) \geq m .
$$

yielding $p_{0}=\bar{p}=1$ contradicting the first inequality in (5.24), so that (5.22) is true. Assume that the first inequality in (5.21) is true, then we can make the choice $(p, w)=\left(t T_{t}^{R}, w^{R y}\right)$ in (5.22) yielding

$$
I\left(t T_{t}^{R} w^{R y}\right) \leq m-\alpha_{0}, \quad I\left((1-t) T_{t}^{R} w^{R x_{0}}\right) \leq m .
$$

Then, using these informations in (5.17), one gets

$$
I_{\Omega}\left(T_{t}^{R} U_{t}^{R}\right) \leq 2 m-\alpha_{0}+O\left(\varepsilon_{R}\right)
$$

and an analogous argument gives the same conclusion when the second inequality in (5.21) holds true. This, Lemma 4.1, and (5.20) yield (5.16) giving the conclusion.

\section{Proof of Theorems 2.2 and 1.1}

Lemma 5.3 yields all the required geometrical properties to apply a Linking Theorem. Moreover from Lemmas 5.2 and 5.4 we deduce that

$$
m_{\Omega} \underset{(a)}{<} d \underset{(b)}{<} 2 m_{\Omega}
$$

Indeed, (b) in (5.25) follows directly from Lemma 5.4 as $h:=i d \in \mathcal{H}$, so that

$$
\inf _{\mathcal{H}} \sup _{Q} I_{\Omega}(h(u)) \leq \sup _{Q} I_{\Omega}(u)<2 m_{\Omega} .
$$

In order to show $(a)$ in (5.25), observe that exploiting property (5.10) we can say that for every $h \in \mathcal{H}$ there exists $v \in Q$ such that $h(v) \in S$, so that

$$
\max _{u \in Q} I_{\Omega}(h(u)) \geq I_{\Omega}(h(v)) \geq \inf _{S} I_{\Omega}>m_{\Omega},
$$

where the last inequality is proved in Lemma 5.2. Corollary 4.6 implies that the (PS) condition is satisfied at level $d$. Then we can apply the Linking Theorem (see e.g. Theorem 8.22 in [5] or Theorem 8.4 in [37] joint with Lemma 5.14 and 5.15 in [39]) to obtain the existence of a constrained critical point of $I$ in $\mathcal{N}_{\Omega}$. This, taking 
into account Remark 2.5, gives the conclusion.

Theorem 1.1 follows directly from Theorem 2.2 as $f$ given in (1.2) satisfies all the requested hypotheses.

Remark 5.5. The previous topological argument shows that the set $\Psi_{R}\left(\partial B_{R}(0)\right)$ is contractible in the sub-level set $I_{\Omega}^{\bar{c}}$ with $\bar{c}$ given by

$$
\bar{c}=\frac{\sup }{B_{R}(0)} I\left(\Psi_{R}\right)
$$

Because the whole set $\Psi_{R}\left(\overline{B_{R}(0)}\right)$ is contractible in $I_{\Omega}^{\bar{c}}$, but $\Psi_{R}\left(\partial B_{R}(0)\right)$ is not contractible in the sub-level set $I_{\Omega}^{\underline{c}}$ for $\underline{c}$ given by

$$
\underline{c}=\max _{\Psi_{R}\left(\partial B_{R}(0)\right)} I .
$$

Moreover, we believe that using appropriate topological tools one can show also multiplicity results depending on the topology of $\Omega$. We leave this topic as an interesting, in our opinion, open problem.

Acknowledgement. Part of this work has been done while the second author was visiting the University of Brasilia. She wishes to thank all the departamento de Matemática for the warm hospitality. A special thank goes to the Liliane's family for the hospitality and the friendly atmosphere.

The authors thank the anonymous referee whose important comments helped them to improve their work.

\section{REFERENCES}

[1] N. Ackermann, M. Clapp and F. Pacella, Alternating sign multibump solutions of nonlinear elliptic equations in expanding tubular domains, Comm. Partial differential equations 38, no. 5 (2013), 751-779.

[2] N. Akhmediev, A. Ankiewicz, Partially coherent solitons on a finite background, Physical Review Letters 82, no. 13, (1999), 2661-2664.

[3] N. Akhmediev, W. Królinowski and A. Snyder, Partially coherent solitons of variable shape, Physical Review Letters 81, no. 21, (1998), 4632-4635.

[4] A. Ambrosetti, G. Cerami and D. Ruiz, Solitons of linearly coupled systems of semilinear nonautonomous equations on $R^{n}$, J. Funct. Anal. 254, no.11, (2008), 2816-2845.

[5] A. Ambrosetti, A. Malchiodi, Nonlinear Analysis and Semilinear Elliptic Problems, Cambridge Stud. Adv. Math., vol. 104, Cambridge Univ. Press, 2006.

[6] A. Bahri, Y.Y. Li, On a min-max procedure for the existence of a positive solution for certain scalar field equations in $\mathbb{R}^{N}$ Rev. Mat. Iberoamericana 6, no. 1/2, (1990), 1-15.

[7] A. Bahri, P.L. Lions, On the existence of a positive solution of semilinear elliptic equations in unbounded domains Ann. Inst. H. Poincaré Anal. Non Linéaire 14, no. 3, (1997), 365-413.

[8] T. Bartsch, T. Weth, Three nodal solutions of singularly elliptic equations on domains without topology, Ann. I. H. Poincaré Anal. Non Linéaire 22, no. 3, (2005), 259-281.

[9] V. Benci, G. Cerami, Positive solutions of some nonlinear elliptic problems in exterior domains, Arch. Rational Mech. Anal. 99, no. 4, (1987), 283-300.

[10] H. Berestycki and P.L. Lions, Nonlinear scalar field equations I and II, Arch. Rational Mech. Anal. 82, no.4, (1983), 313-345 and 347-375.

[11] H. Berestycki and T. Gallouet, O. Kavian Équations de champs scalaires euclidiens non linéaires dans le plan, C. R. Acad. Sci. Paris 297, série I, (1983), 307-310. 
[12] H. Brezis, E. Lieb, A relation between pointwise convergence and convergence of functionals, Proceedings of the American Mathematical Society 88, no.3, (1983), 486-490.

[13] G. Cerami, Un criterio di esistenza per i punti critici su varietà illimitate, Rend. Accad. Sc. Lett. Inst. Lombardo 112, (1978), 332-336.

[14] G. Cerami, Some nonlinear elliptic problems in unbounded domains, Milan J. Math. 74, (2006), 47-77.

[15] G. Cerami and M. Clapp, Sign-changing solutions of semilinear elliptic problems in exterior domains, Calc. Var. Partial Differential Equations 30, no. 3, (2007), 353-367.

[16] G. Cerami, R. Molle, D. Passaseo, Positive solutions of semilinear elliptic problems in unbounded domains with unbounded boundary, Ann. I. H. Poincaré AN. 24, no. 1, (2007), 41-60.

[17] G. Cerami, D. Passaseo, Existence and multiplicity of positive solutions for nonlinear elliptic problems in exterior domains with "rich" topology, Nonlinear Analysis TMA 18, no. 2, (1992), $109-119$.

[18] G. Cerami, D. Passaseo, Existence and multiplicity results for semi linear elliptic dirichlet problems in exterior domains, Nonlinear Analysis TMA 24, no.11, (1995), 1533-1547.

[19] G. Citti, On the exterior Dirichlet problem for $\Delta u-u+f(x, u)=0$. Rendiconti del seminario matematico dell'università di Padova 88 (1992), 83-110.

[20] M. Clapp, D. Salazar, Multiple sign changing solutions of nonlinear elliptic problems in exterior domains. Advanced Nonlinear Studies 12, no.3, (2012), 427-443.

[21] D. Costa, C.A. Magalhaes, Variational elliptic problems which are non quadratic at infinity, Nonlinear Analysis TMA 23, no. 11, (1994), 1401-1412.

[22] M. Esteban, P.L. Lions, Existence and nonexistence results for semilin-ear elliptic problems in unbounded domains, Proc. Roy. Soc. Edinburgh Sect. A 93, no. 1-2, (1982), 1-14.

[23] G. Evéquoz, T. Weth, Entire solutions to nonlinear scalar field equations with indefinite linear part, Adv. Nonlinear Stud. 12 (2012), no. 2, 281-314

[24] G.Li, G. Zheng, The existence of positive solution to some asymptotically linear elliptic equations in exterior domains, Rev. Mat. Iberoamericana 22, no.2, (2006), 559-590.

[25] P.L. Lions, The concentration-compactness principle in the calculus of variations. The locally compact case, Ann. I. H. Poincaré, A. N. 1, no. 1-2 (1984), 109-145 and 223-283.

[26] N.M. Litchinitser, W. Królikowski, N.N. Akhmediev, G.P. Agrawal, Asymmetric partially coherent solitons in saturable nonlinear media, Phys. Rev. E 60 (1999), 2377-2380.

[27] L.A. Maia, O.H. Miyagaki, S.H.M. Soares, Sign-changing solution for an asymptotically linear Schrödinger equation. Proc. Edin. Mathematical Society 58, no.3, (2015), 697-716.

[28] L. Maia, E. Montefusco, B. Pellacci, Weakly coupled nonlinear Schrödinger systems: the saturation effect, Calc. Var. Partial Differential Equations 46, no.1-2, (2013), 325-351.

[29] Z. Nehari, Characteristic values associated with a class of non-linear second-order differential equations, Acta Math. 105 (1961), 141-175.

[30] Z. Nehari, A nonlinear oscillation theorem, Duke Math. J. 42 (1975), 183-189.

[31] B. Noris, G. Verzini A remark on natural constraints in variational methods and an application to superlinear Schrödinger systems, J. differential equations 254, no. 3, (2013), 1529-1547.

[32] E.A. Ostrovskaya, Y.S. Kivshar, Multi-hump optical solitons in a saturable medium, J. Opt. B: Quantum Semiclass. Opt. 1 (1999), 77-83.

[33] P.H. Rabinowitz, On a class of nonlinear Schrödinger equations, Z. Angew. Math. Phys. 43 (1992) 270-291.

[34] J. Serrin, M. Tang, Uniqueness of ground states for quasilinear elliptic equations, Indiana Univ. Math. J. 49 (2000), 897-923.

[35] G.I. Stegeman, D. N. Christodoulides, M. Segev, Optical spatial solitons: Historical Perspectives, IEEE Journal on selected topics in quantum electronics 6 (2000), 1419-1427.

[36] C.A. Stuart, H.S. Zhou, Applying the mountain pass theorem to an asymptotically linear elliptic equation on $\mathbb{R}^{N}$, Comm. Partial Differential Equations 24, no.9-10 (1999),1731-1758.

[37] M. Struwe, Variational Methods, Volume 34, Springer, Berlin, 2008.

[38] A. Szulkin, T. Weth, The method of Nehari manifold. Handbook of nonconvex analysis and applications, 597-632, Int. Press, Somerville, MA, 2010.” 
[39] M. Willem, Minimax theorems, Progress in Nonlinear Differential Equations and their Applications 24, Birkhäuser Boston, 1996.

(L. A. Maia) Departmento de Matemática, Universidade de Brasília, 70910-900 Brasilia, Brazil. E-mail address, L.A. Maia: lilimaia@unb.br

(B. Pellacci) Dipartimento di Scienze e Tecnologie, Università di Napoli "Parthenope", Centro Direzionale, Isola C4 80143 Napoli, Italy.

E-mail address, B. Pellacci: benedetta.pellacci@uniparthenope.it 